\title{
How Many Diagonal Rectangles Are Needed to Cover an Orthogonal Polygon?*
}

\author{
Yoshiyasu Ishigami \\ Department of Information and Communication Engineering, \\ The University of Electro-Communications, Tokyo 182-8585, Japan
}

\begin{abstract}
We show that any orthogonal polygon of $n$ vertices can be covered with at most $\left\lfloor\frac{3}{4} n\right\rfloor-2-\omega$ "diagonal rectangles" where $\omega=1(n=8,12,16)$ and $\omega=0$ (otherwise). An orthogonal polygon is a polygon whose edges are horizontal or vertical. A diagonal rectangle (of an orthogonal polygon) is a rectangle whose opposite corners are vertices of the orthogonal polygon. The result is sharp and settles a question of Mamoru Watanabe [11].
\end{abstract}

\section{Introduction}

In this paper we consider a problem of covering orthogonal polygons with rectangles. Needless to say, orthogonal polygons are one of the most important and fundamental subclasses of polygons, arisen naturally in domains such as VLSI design, pattern recognition, or architecture. Orthogonal polygons have been also called "rectilinear polygons" or "polyominoes" in some papers and books (see the footnote on p. 31 of [10]). Problems of covering orthogonal polygons with rectangles have numerous theoretical and practical applications within and without computer science (see, e.g., [4]).

First, we define some words formally. A (holeless) polygon is a closed connected region of the plane enclosed by a simple cycle of straight line segments where a simple cycle means that nonconsecutive segments do not intersect and two consecutive segments intersect only at their common endpoint. An orthogonal polygon is a polygon such that the line segments of the boundary (called edges) are vertical or horizontal. See Fig. 1. We call an endpoint of an edge a vertex. Clearly the number of vertices is equal

\footnotetext{
* This research was partially supported by the Japan Society for the Promotion of Science, and by the Ministry of Education, Science, Sports and Culture, Grant-in-Aid for Encouragement of Young Scientists. The author is at the Department of Mathematics, University of Illinois at Urbana-Champaign, 1409 W. Green Street, Urbana, IL 61801-2975,USA from April 1999 to March 2001. yoshiyas@uiuc.edu.
} 


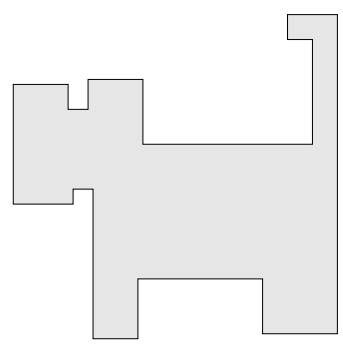

Fig. 1. An orthogonal polygon.

to the number of edges. An orthogonal polygon of four edges is called a rectangle (or a box).

A family $\mathcal{C}$ of rectangles covers an orthogonal polygon $P$ if and only if the union of the rectangles equals $P$, i.e., $\bigcup_{R \in \mathcal{C}} R=P$. We allow overlappings of rectangles but not rotation. The following proposition gives the worst-case number of rectangles necessary to cover an orthogonal polygon.

Proposition 1. Every orthogonal polygon of $n$ vertices can be covered with at most $\lfloor n / 2\rfloor-1$ rectangles. Moreover it is sharp.

Although it is quite easy to prove, the proof is presented in Section 3 because it provides a good warm-up exercise before we start the proof of the main theorem.

The topic has practical applications and has been researched in computer science and combinatorics since the late 1970s. When holes in orthogonal polygons are allowed, the problem of minimally covering orthogonal polygons with rectangles was shown to be NP-complete by Masek [9]. Although the holeless case had been long-standing, in 1994 Culberson and Reckhow [3] showed that the problem is NP-complete for holeless orthogonal polygons. From a holeless or nonholeless orthogonal polygon $P$, we can define a hypergraph $H(P)=(V(P), E(P))$ by $V(P)=P$ and $E(P)=\{E \subset P \mid E$ is a rectangle $\}$. Chvátal first asked whether $\theta(P) / \alpha(P)=1$ for all $P$ where $\theta(P)$ or $\alpha(P)$ is the covering number (i.e., the minimum number of edges covering the vertices) or the independence number of $H(P)$, respectively. After this was disproved by Szemerédi and Chung, Erdôs asked if $\theta(H(P)) / \alpha(H(P))$ is bounded. This still remains open. See [2]. Meanwhile Berge et al. [1] and Győri [5] studied this problem for special types of orthogonal polygons (for example, horizontally convex orthogonal polygons). Other previous researches have focused on finding fast algorithms for special types of orthogonal polygons. See [4] for details.

In this paper we do not restrict the shape of the orthogonal polygon which we try to cover but we restrict rectangles available to cover the polygon. A rectangle $R$ is a diagonal rectangle (of an orthogonal polygon $P$ ) if and only if, for at least one of the two diagonal lines of $R$, the endpoints of it are both vertices of $P$ (See Fig. 2). We say that a family $\mathcal{C}$ of rectangles (or diagonal rectangles) is a rectangle cover (or diagonal-rectangle cover) of $P$ if it covers $P$. This restriction of rectangles seems to be very natural and has applications. In most situations, a rectangle is usually defined 

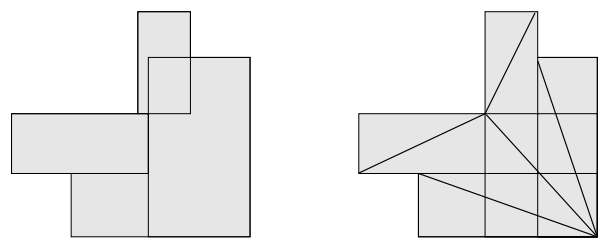

Fig. 2. An orthogonal polygon covered with four rectangles or with five diagonal rectangles.

by choosing a pair of diagonal points of it. Another type of restriction of rectangles was discussed in [8]. Mamoru Watanabe [11] proposed the problem of minimally covering orthogonal polygons with diagonal rectangles. Our goal is to prove the following main theorem.

Theorem 1. Every orthogonal polygon of $n$ vertices can be covered with at most $\left\lfloor\frac{3}{4} n\right\rfloor-$ $2-\omega$ "diagonal rectangles" where $\omega=1(n=8,12,16)$ and $\omega=0$ (otherwise). Moreover it is sharp.

In Section 2 we show the sharpness of the proposition and the theorem. Then, in Section 3, we prove the proposition as a warm-up. Most definitions and all lemmas in these sections are also used later for the proof of the theorem. We prepare more definitions and lemmas in Section 4. Finally, we prove the theorem in Section 5.

Our proof strategy is to investigate neighborhoods of local structures called "tabs." They were first introduced by Kahn et al. [7], [10] in order to prove the well-known art gallery theorem of orthogonal polygons. Györi [6] found a shorter proof of the theorem with a different technique.

Since nonorthogonal polygons do not appear until the end of this paper, we often shorten "orthogonal polygon" to "polygon."

\section{Examples}

There are two types of vertices, i.e., convex vertices (those with interior angle $90^{\circ}$ ) and reflex vertices (those with interior angle $270^{\circ}$ ). We give a basic relationship about the number $r$ of reflex vertices. For example, the polygon shown in Fig. 1 has nine reflex vertices. Throughout this paper, our discussion is phrased in terms of $r$ for convenience.

Lemma 1. For any orthogonal polygon $P$ of $n$ vertices, it holds that $n=2 r+4$ where $r$ is the number of the reflex vertices.

Proof. Let $c$ be the number of the convex vertices. Clearly, $n=c+r$. Since the sum of the internal angles of a polygon of $m$ vertices is $(m-2) \pi$, the sum of the internal angles of $P$ is $(n-2) \pi$. It is also equal to $c(\pi / 2)+r(3 \pi / 2)=(n / 2+r) \pi$. The equality $n-2=n / 2+r$ yields the desired equation. 


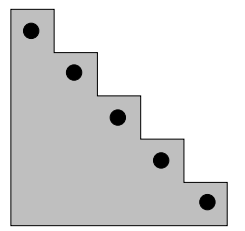

Fig. 3. An orthogonal polygon showing the sharpness of Proposition 1.

By Lemma 1, Proposition 1 and Theorem 1 can be rewritten with term $r$ as follows.

Proposition 1'. Every orthogonal polygon of $r$ reflex vertices can be covered with at most $r+1$ rectangles.

Theorem 1'. Every orthogonal polygon of $r$ reflex vertices can be covered with at most $\left\lfloor\frac{3}{2} r\right\rfloor+1-\omega$ diagonal rectangles, where $\omega=1(r=2,4,6)$ and $\omega=0$ (otherwise). Moreover it is sharp.

For the sharpness of Proposition 1, see Fig. 3. Note that in Fig. 3 any pair of the $r+1$ black points cannot be covered with only one rectangle simultaneously. Thus $r+1$ rectangles are necessary to cover the example.

Next we see the sharpness of Theorem 1. See Fig. 4. We can observe that in Fig. 4 any pair of the black points cannot be covered with only one diagonal rectangle simultaneously. We call such a set of black points independent points and the maximal size of such points is called the independence number of the polygon. It is clear that a polygon needs more diagonal rectangles to be covered than the independence number. Any polygon in Fig. 4 contains the desired number of independent points. Therefore the examples show the sharpness of Theorems 1 and $1^{\prime}$. It is clear that we can construct an example for $r \geq 16$ in the same way.

Obviously there are many other examples showing the sharpness. For example, we can also make an example for $r=9,11,13$ or for $r \geq 15$ by "connecting" an example of $r=1,3,5,7,8,10,12,14$ and several examples of $r=8$. See Fig. 5 .

\section{Proof of Proposition 1}

The purpose of this section is not only to prove the proposition but also to give some definitions and a basic property.

Throughout this paper we write the horizontal or vertical coordinate of vertex $v$ by $(v)_{\mathrm{H}}$ or $(v)_{\mathrm{V}}$, respectively. We may write $v$ by vector form $v=\left((v)_{\mathrm{H}},(v)_{\mathrm{V}}\right)$ where we take the coordinate axes toward the top and right. For $i \in\{H, V\}$, define the $i$-distance between vertices $u, v$ by

$$
\operatorname{dist}_{i}(u, v):=\left|(u)_{i}-(v)_{i}\right|
$$

(we sometimes use the symbol ":=" instead of "=" when defining the left-hand side by 


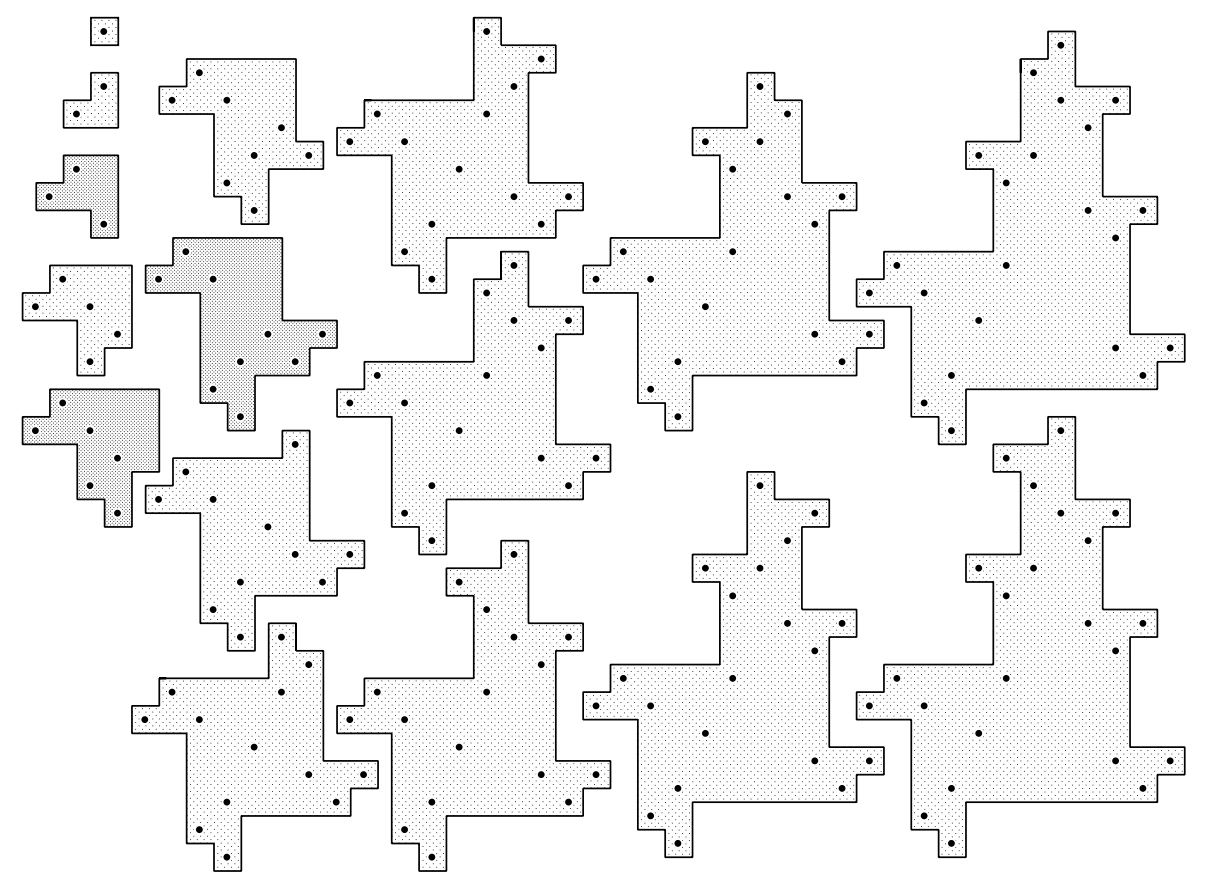

Fig. 4. Examples for $r=0,1, \ldots, 15$.

the right-hand side). The $\mathrm{V}$-distance $\operatorname{dist}_{\mathrm{V}}\left(e, e^{\prime}\right)$ (or H-distance $\operatorname{dist}_{\mathrm{H}}\left(e, e^{\prime}\right)$ ) between two horizontal (or vertical) edges $e, e^{\prime}$ are defined naturally. For $i=\mathrm{H}, \mathrm{V}$, if $(u)_{i}<(v)_{i}$, then we write $u<_{i} v$ or say $u$ is horizontally left of $v$. For any point $p$ in $P$, let $u p(p)$ be the highest point $q \in P$ such that segment $p q$ is vertical and contained completely in $P$. We also define $>_{i},=_{i}, \leq_{i}, \geq_{i}, \operatorname{down}(p), \operatorname{right}(p)$, and left $(p)$ similarly. Furthermore, for a horizontal edge $e$ and a vertical edge $f, \operatorname{left}(e), \operatorname{right}(e), \operatorname{up}(f)$, and $\operatorname{down}(f)$ are defined naturally. For example, $p=\operatorname{down}(u)=\operatorname{down}(f)$ in Fig. 7 where $u$ is an end vertex of vertical edge $f$.

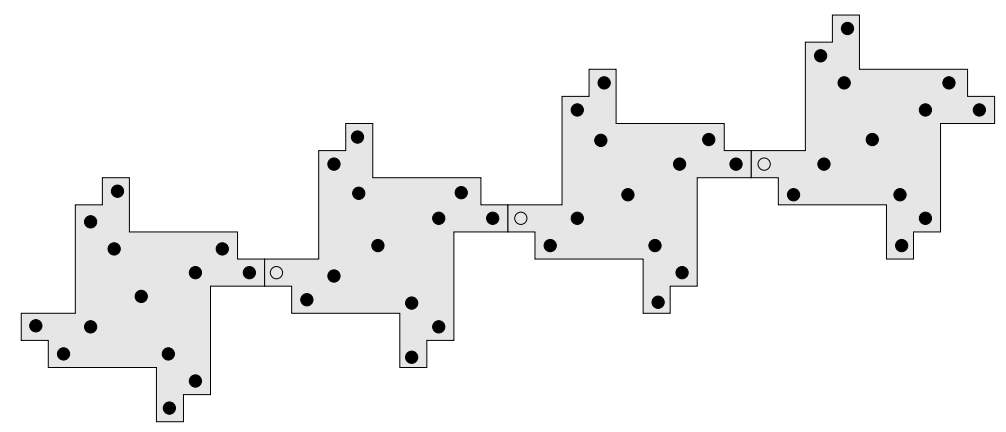

Fig. 5. An example of $r=8+8 \times 3=32$. 
For a polygon $P$, let $V(P)$ or $E(P)$ be the set of vertices or edges, respectively. For vertices $u, v$, we denote the (closed) line segment whose endpoints are $u$ and $v$ by $u v$. It is clear that from $V(P)$ and $E(P), P$ can be determined uniquely. Define an orthogonal polygon to be in general integer position if (i) for any two nonadjacent vertices, $\operatorname{dist}_{\mathrm{H}}(u, v)>0$ and $\operatorname{dist}_{\mathrm{V}}(u, v)>0$, and (ii) for each vertex $u,(u)_{\mathrm{H}}$ and $(u)_{\mathrm{V}}$ are integers.

The following lemma is not essential for our proofs but it gives a basic fact in this kind of topic (for example, see Lemma 2.1 in [10]). See Section 6.

Lemma 2. If any orthogonal polygon of $r$ reflex vertices in general integer position can be covered with $m$ rectangles (or with $m^{\prime}$ diagonal rectangles), then any orthogonal polygon of $r$ reflex vertices can be covered with $m$ rectangles (or with $m^{\prime}$ diagonal rectangles, respectively).

Proof. Let $Q$ be an orthogonal polygon of $r$ reflex vertices that is or is not in general integer position. We consider making a new polygon $Q^{\star}$ by "moving" an edge "a little." For real number $\varepsilon$ and a horizontal edge $e=u v$, if

$$
\left\{w \in V(Q) \mid(u)_{\mathrm{V}}<(w)_{\mathrm{V}}<(u)_{\mathrm{V}}+\varepsilon\right\}=\emptyset,
$$

then there exists an orthogonal polygon $Q^{\star}=Q+{ }_{e} \varepsilon$ with the same $r$ such that $V\left(Q^{\star}\right)=$ $(V(Q)-\{u, v\}) \cup\left\{u^{\star}, v^{\star}\right\}$ and $E\left(Q^{\star}\right)=\left(E(Q)-\left\{u^{\prime} u, u v, v v^{\prime}\right\}\right) \cup\left\{u^{\prime} u^{\star}, u^{\star} v^{\star}, v^{\star} v^{\prime}\right\}$ where $u^{\star}:=u+\varepsilon(0,1), v^{\star}:=v+\varepsilon(0,1)$ and $u^{\prime}, v^{\prime}$ are the vertices adjacent to $u$ and $v$, respectively. See Fig. 6 . It is clear that if $Q^{\star}$ is covered with some rectangles (or diagonal rectangles), then $Q$ can be covered with at most the same number of rectangles (or diagonal rectangles, respectively) by deleting some old rectangles and adding some new rectangles. For $\varepsilon$ and a vertical edge $e$, polygon $P^{\star}=P+_{e} \varepsilon$ is defined and has the same property similarly.

By using the above observation repeatedly, we complete the proof. Let $P$ be an orthogonal polygon of $r$ reflex vertices that is not in general integer position. Clearly, $P$ has $n$ vertices and $n$ edges for $n=2 r+4$ because of Lemma 1 . Without loss of generality, we can assume that

$$
\operatorname{dist}_{i}(u, v)=0 \text { or }>1000 n \quad \text { for any } \quad u, v \in V(P) \quad \text { and } \quad i=\mathrm{H}, \mathrm{V}
$$

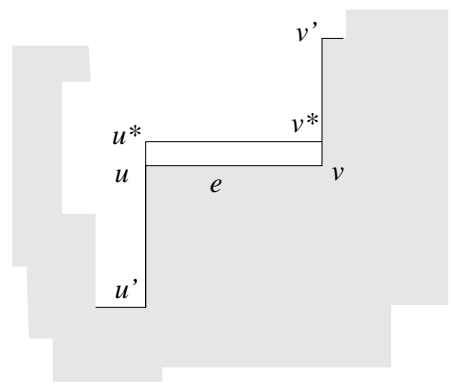

Fig. 6. Definition of $Q^{\star}=Q+e \varepsilon$. 


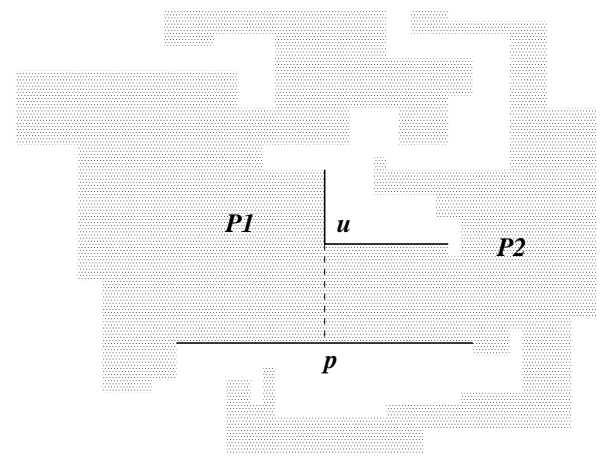

Fig. 7. Proof of Proposition 1.

(enlarge the sheet). For every edge $e_{i}(i=1, \ldots, n)$, we repeat the above " $+{ }_{e} \varepsilon$ "procedure for all edges. Let $e_{1}=u_{1} v_{1}, \ldots, e_{n}=u_{n} v_{n}$ be all of the edges and put $\varepsilon_{i}=10(n-i)+\left\lceil\left(u_{i}\right)_{j}\right\rceil-\left(u_{i}\right)_{j}(i=1, \ldots, n)$ where $j=\mathrm{V}$ (or $\left.j=\mathrm{H}\right)$ if and only if $e_{i}$ is horizontal (or vertical, respectively). We define polygon $P^{\star n}:=\left(\cdots\left(\left(P+e_{e_{1}}\right.\right.\right.$ $\left.\left.\left.\varepsilon_{1}\right)+{ }_{e_{2}} \varepsilon_{2}\right) \cdots\right)+{ }_{e_{n}} \varepsilon_{n}$. Note that because of (2) property (1) is always valid. Therefore the polygon $P^{\star n}$ is well defined. It is clear that $P^{\star n}$ is in general integer position. Due to the above observation about $Q$ and $Q^{\star}$, if $P^{\star n}$ is covered with some rectangles (or diagonal rectangles), then $P$ can be covered with at most the same number of rectangles (or diagonal rectangles).

Now we prove Proposition 1 very quickly.

Proof of Proposition 1. We prove by induction on $r$. It is trivial for $r=0$. Without loss of generality, there is a reflex vertex $u$ such that $u+(0.5,0.5) \notin P$. See Fig 7 . By Lemma 2, we can assume that $P$ is in general integer position. Thus $p:=\operatorname{down}(u)$ is not a vertex. We cut polygon $P$ by segment $u p$, which yields two polygons $P_{i}(i=1,2)$ having $r_{i}$ reflex vertices with $r_{1}+r_{2}+1=r$. By the induction hypothesis, $P_{i}$ has a rectangle cover $\mathcal{C}_{i}$ of size $r_{i}+1$. Then $\mathcal{C}_{1} \cup \mathcal{C}_{2}$ covers $P$ and $\left|\mathcal{C}_{1} \cup \mathcal{C}_{2}\right| \leq r_{1}+r_{2}+2=$ $r+1$.

\section{Definitions and Preliminary Lemmas}

We introduced basic terminology and lemmas in the above sections. We prepare more technical terms and lemmas to prove the main theorem smoothly.

Let $P$ be an orthogonal polygon of $r$ reflex vertices in general integer position. Let $e$ be a horizontal edge. There exists exactly one vertical edge $f$ containing point left $(e)$. We call it the left facing edge of $e$. See Fig. 8(a). If the left end vertex of $e$ is convex, then left $(e)$ equals the end vertex and $e$ and the left facing edge $f$ are adjacent. The right facing edge is defined similarly. 


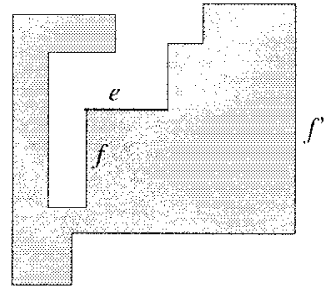

(a)

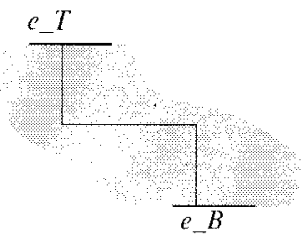

(b)

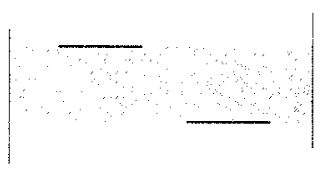

(c)

Fig. 8. (a) The left and right facing edges $f$ and $f^{\prime}$ of $e$, (b) top edge $e_{\mathrm{T}}$ and bottom edge $e_{\mathrm{B}}$ see one another, and (c) a pair of neighboring edges.

It is said that a horizontal edge $e$ is a top edge (or bottom edge) if and only if the interior of $P$ lies below (or above) it; left and right edges are defined similarly. Note that left (or right) facing edges are always left (or right) edges. A top edge $e_{\mathrm{T}}$ sees a bottom edge $e_{\mathrm{B}}$ if and only if there exist points $p_{\mathrm{T}} \in e_{\mathrm{T}}, p_{\mathrm{B}} \in e_{\mathrm{B}}$ and real number $s\left(\left(p_{\mathrm{T}}\right)_{\mathrm{V}}>s>\left(p_{\mathrm{B}}\right)_{\mathrm{V}}\right)$ such that

(i) three line segments $p_{\mathrm{T}}\left(\left(p_{\mathrm{T}}\right)_{\mathrm{H}}, s\right),\left(\left(p_{\mathrm{T}}\right)_{\mathrm{H}}, s\right)\left(\left(p_{\mathrm{B}}\right)_{\mathrm{H}}, s\right),\left(\left(p_{\mathrm{B}}\right)_{\mathrm{H}}, s\right) p_{\mathrm{B}}$ are all contained in $P$, and

(ii) $\left(p_{\mathrm{T}}\right)_{\mathrm{H}}-\left\lfloor\left(p_{\mathrm{T}}\right)_{\mathrm{H}}\right\rfloor=\left(p_{\mathrm{B}}\right)_{\mathrm{H}}-\left\lfloor\left(p_{\mathrm{B}}\right)_{\mathrm{H}}\right\rfloor=s-\lfloor s\rfloor=0.5$.

See Fig. 8(b). If top $e_{\mathrm{T}}$ sees bottom $e_{\mathrm{B}}$, then $e_{\mathrm{T}}$ is higher than $e_{\mathrm{B}}$. We say that bottom edge $e_{\mathrm{B}}$ also sees top edge $e_{\mathrm{T}}$ when $e_{\mathrm{T}}$ sees $e_{\mathrm{B}}$. The nearest visible edge nv(e) from $e$ is the edge seeing $e$ such that, for any horizontal edge $e^{\prime}$ with $\operatorname{dist}_{\mathrm{V}}\left(e, e^{\prime}\right)<\operatorname{dist}_{\mathrm{V}}(e, \operatorname{nv}(e))$, $e^{\prime}$ does not see $e$. Because $P$ is in general integer position, every horizontal edge has its unique nearest visible one. If a top edge $e$ and a bottom edge $e^{\prime}$ are the nearest visible edges from one another, we say that $e$ and $e^{\prime}$ are neighboring.

For any two points $p, q$, we let $\square(p, q)$ denote the unique rectangle defined by diagonal $p q$, i.e., $\square(p, q):=\left\{\left(s_{\mathrm{H}}, s_{\mathrm{V}}\right) \in \mathbf{R}^{2} \mid \min \left\{(p)_{i},(q)_{i}\right\} \leq s_{i} \leq \max \left\{(p)_{i},(q)_{i}\right\}\right.$ for each $i=\mathrm{H}, \mathrm{V}\}$.

The following is the most basic property of a pair of neighbors.

Lemma 3. Let $P$ be an orthogonal polygon in general integer position. Then

(i) $P$ has a pair of neighboring edges, and

(ii) if $e_{\mathrm{T}}$ and $e_{\mathrm{B}}$ are top and bottom neighboring edges, then the left and right facing edges of $e_{T}$ equal the left and right facing edges of $e_{\mathrm{B}}$, respectively, and the rectangle enclosed by $e_{\mathrm{T}}, e_{\mathrm{B}}$ and the two facing edges is contained in $P$, i.e., $\square\left(\operatorname{left}\left(e_{\mathrm{T}}\right), \operatorname{right}\left(e_{\mathrm{B}}\right)\right) \subset P$ (see Fig. 8(c)).

Proof. (i) Take a top edge $e$ of $P$ and its nearest visible edge nv $(e)$. Bottom edge nv $(e)$ also has its nearest visible edge $\operatorname{nv}(\operatorname{nv}(e))=\mathrm{nv}^{2}(e)$. Since the number of edges is finite, there exist integers $i$ and $m$ such that $n v^{i}(e)=n v^{i+2 m}(e)$. See Fig. 9(a). If $m>1$, then $\operatorname{dist}_{\mathrm{V}}\left(\mathrm{nv}^{i}(e), \mathrm{nv}^{i+2 m-1}(e)\right)<\operatorname{dist}_{\mathrm{V}}\left(\mathrm{nv}^{i}(e), \mathrm{nv}^{i+1}\right)$ and $\mathrm{nv}^{i}(e)=\mathrm{nv}^{i+2 m}(e)$ sees $\mathrm{nv}^{i+2 m-1}(e)$, which contradicts the definition of $\mathrm{nv}^{i+1}(e)$. Then $m=1$, meaning that $n v^{i}(e)=\mathrm{nv}^{i+2}(e)$. Therefore we have a pair of neighboring edges $\mathrm{nv}^{i}(e)$ and $\mathrm{nv}^{i+1}(e)$. 


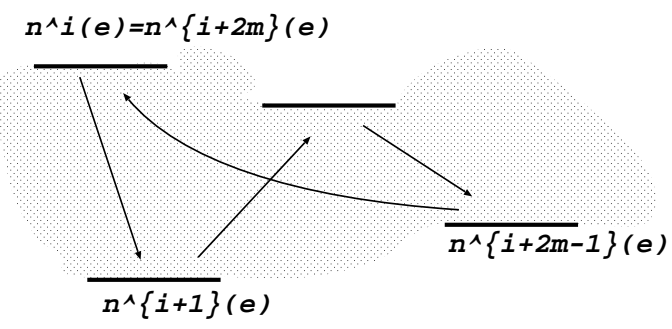

(a)

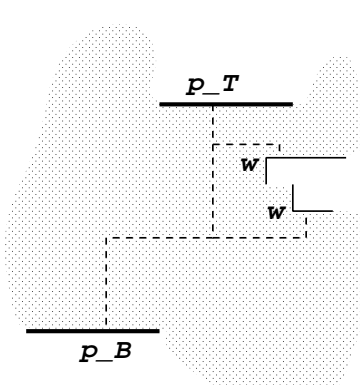

(b)

Fig. 9. Proof of Lemma 3 (a) for (i) and (b) for (ii).

(ii) Since $e_{\mathrm{T}}$ sees $e_{\mathrm{B}}$, there are points $p_{\mathrm{T}} \in e_{\mathrm{T}}, p_{\mathrm{B}} \in e_{\mathrm{B}}$ and real number $s\left(\left(p_{\mathrm{T}}\right)_{\mathrm{V}}>\right.$ $\left.s>\left(p_{\mathrm{B}}\right)_{\mathrm{V}}\right)$ such that (a) three line segments $p_{\mathrm{T}}\left(\left(p_{\mathrm{T}}\right)_{\mathrm{H}}, s\right),\left(\left(p_{\mathrm{T}}\right)_{\mathrm{H}}, s\right)\left(\left(p_{\mathrm{B}}\right)_{\mathrm{H}}, s\right)$, $\left(\left(p_{\mathrm{B}}\right)_{\mathrm{H}}, s\right) p_{\mathrm{B}}$ are all contained in $P$, and $(\mathrm{b})\left(p_{\mathrm{T}}\right)_{\mathrm{H}}-\left\lfloor\left(p_{\mathrm{T}}\right)_{\mathrm{H}}\right\rfloor=\left(p_{\mathrm{B}}\right)_{\mathrm{H}}-\left\lfloor\left(p_{\mathrm{B}}\right)_{\mathrm{H}}\right\rfloor=$ $s-\lfloor s\rfloor=0.5$. Let $W:=\left\{w \in V(P) \mid\left(p_{\mathrm{T}}\right)_{\mathrm{V}}>(w)_{\mathrm{V}}>s\right.$ and $\left.w\left(\left(p_{\mathrm{T}}\right)_{\mathrm{H}},(w)_{\mathrm{V}}\right) \subset P\right\}$. Without loss of generality, by way of contradiction, we can assume that $W \neq \emptyset$. Let $w \in W$ be a leftmost vertex in $W$. (Since $P$ is in general integer position, there is one or two such $w$ 's.) By the definition of $w, w$ must be reflex such that $w+(-0.5, \pm 0.5) \in P$. See Fig. 9(b).

If $w+(0.5,-0.5) \notin P$, then it is clear that three line segments $p_{\mathrm{T}}\left(\left(p_{\mathrm{T}}\right)_{\mathrm{H}},(w)_{\mathrm{V}}+\right.$ $0.5),\left(\left(p_{\mathrm{T}}\right)_{\mathrm{H}},(w)_{\mathrm{V}}+0.5\right)\left((w)_{\mathrm{H}}+0.5,(w)_{\mathrm{V}}+0.5\right),\left((w)_{\mathrm{H}}+0.5,(w)_{\mathrm{V}}+0.5\right)\left((w)_{\mathrm{H}}+\right.$ $\left.0.5,(w)_{\mathrm{V}}\right)$ are all contained in $P$, yielding that the bottom edge incident to $w$ is seen by $e_{\mathrm{T}}$ and $\operatorname{dist}_{\mathrm{V}}\left(e_{\mathrm{T}}, w\right)<\operatorname{dist}_{\mathrm{V}}\left(e_{\mathrm{T}}, e_{\mathrm{B}}\right)$, a contradiction.

Let $w+(0.5,0.5) \notin P$. Consider three line segments $(w+(0.5,0))\left((w)_{\mathrm{H}}+0.5, s\right)$, $\left((w)_{\mathrm{H}}+0.5, s\right)\left(\left(p_{\mathrm{B}}\right)_{\mathrm{H}}, s\right)$, and $\left(\left(p_{\mathrm{B}}\right)_{\mathrm{H}}, s\right) p_{\mathrm{B}}$. They are all in $P$, which means that the top edge incident to $w$ is seen by $e_{\mathrm{B}}$ and $\operatorname{dist}_{\mathrm{V}}\left(e_{T}, w\right)<\operatorname{dist}_{\mathrm{V}}\left(e_{T}, e_{\mathrm{B}}\right)$, a contradiction.

Let $\mathcal{C}$ be a class of diagonal rectangles. We denote the set of vertices used in $\mathcal{C}$ by $V(\mathcal{C})$ that is,

$$
V(\mathcal{C}):=\{u \in V(P) \mid \square(u, v) \in \mathcal{C} \text { for some } v \in V(P)\} .
$$

When a pair of neighboring edges is connected by a vertical left edge, there are four cases as in Fig. 10. Note that property (ii) in Lemma 3 suggests that there are no other vertices between $e_{\mathrm{T}}$ and $e_{\mathrm{B}}$. The following lemma is trivial but useful throughout the main proof.

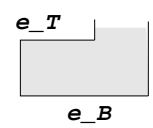

(a)

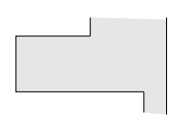

(b)

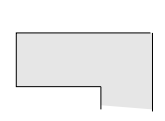

(c)

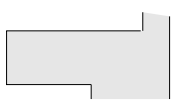

(d)

Fig. 10. Pairs of neighboring edges connected by a vertical edge. 
Lemma 4. Let $P$ be an orthogonal polygon in general integer position. Let top edge $e_{\mathrm{T}}=a b\left(a<_{\mathrm{H}} b\right)$ and bottom edge $e_{\mathrm{B}}=c d\left(c<_{\mathrm{H}} d\right)$ form a neighboring pair connected by left edge ac with $a=_{\mathrm{H}} c<_{\mathrm{H}} b<_{\mathrm{H}} d$ (see Fig. 10(a),(b)). If $P$ is covered with $m$ diagonal rectangles, then $P$ also has a diagonal-rectangle cover $\mathcal{C}$ of size at most $m$ such that $c \notin V(\mathcal{C}), \square(a, d) \in \mathcal{C}$, and $\square(a, v) \notin \mathcal{C}$ for any $v \in V(P)-d$.

Proof. Let $\mathcal{C}$ be a diagonal-rectangle cover of size $m$. To cover point $c+(0.5,0.5), \mathcal{C}$ must have $\square(a, d)$ or $\square(b, c)$. Since $\square(b, c) \subset \square(a, d), \mathcal{C}^{\prime}:=\mathcal{C} \cup\{\square(a, d)\}-\square(b, c)$ is also a diagonal-rectangle cover of size $m$ or $m-1$. For any $v \in V(P), \square(c, v)$ is a diagonal rectangle contained in $P$ then $v=b$ because of Lemma 3(ii). Therefore, $c \notin V\left(\mathcal{C}^{\prime}\right)$. Clearly, $\square(a, v) \notin \mathcal{C}$ for any $v \in V(P)-d$.

The following lemma is one of the keys of the main proof. By combining Lemmas 4 and 5, we can replace and reposition rectangles more freely.

Lemma 5. Let $P$ be an orthogonal polygon of $r$ reflex vertices in general integer position. We also let $r$ be even and a neighboring pair of top $e_{\mathrm{T}}=a b\left(a<_{\mathrm{H}} b\right)$ and bottom $e_{\mathrm{B}}=c d\left(c<_{\mathrm{H}} d\right)$ connected by left ac with $a=_{\mathrm{H}} c<_{\mathrm{H}} b<_{\mathrm{H}} d$ (see Fig. 10). Suppose that any orthogonal polygon of $r+1$ reflex vertices can be covered with $m=\lfloor 3(r+1) / 2\rfloor+1$ diagonal rectangles. Then $P$ (of $r$ reflex vertices in general integer position) can be covered with at most $m-1=\lfloor 3 r / 2\rfloor+1$ diagonal rectangles $\mathcal{C}$ such that $a \notin V(\mathcal{C}), \square(b, c) \in \mathcal{C}$, and $\square(c, v) \notin \mathcal{C}$ for any $v \in V(P)-b$.

Proof. Let $P^{\prime}:=P-\square\left(c, c^{\prime}\right)$ be covered with $m$ diagonal rectangles $\mathcal{C}$ where $c^{\prime}=c+(0.5,0.5)$. Since $\mathcal{C}$ covers points $c+\left(\frac{1}{4}, \frac{3}{4}\right)$ and $c+\left(\frac{3}{4}, \frac{1}{4}\right), \mathcal{C} \cap\{\square(c+$ $\left.(0,0.5), b), \square\left(a, c^{\prime}\right)\right\} \neq \emptyset$ and $\mathcal{C} \cap\left\{\square\left(c^{\prime}, d\right), \square(c+(0.5,0), b)\right\} \neq \emptyset$. We can assume that $\mathcal{C} \supset\left\{\square(c+(0,0.5), b), \square\left(c^{\prime}, d\right)\right\}$ and $\mathcal{C} \cap\left\{\square\left(a, c^{\prime}\right), \square(c+(0.5,0), b)\right\}=\emptyset$ because $\square(c+(0,0.5), b) \cup \square\left(c^{\prime}, d\right) \supset \square(c+(0,0.5), b) \cup \square\left(a, c^{\prime}\right) \cup \square\left(c^{\prime}, d\right) \cup \square(c+(0.5,0), b)$. We let $\mathcal{C}^{\prime}:=\mathcal{C} \cup\{\square(b, c)\}-\left\{\square(c+(0,0.5), b), \square\left(c^{\prime}, d\right)\right\}$. Polygon $P$ is in general integer position and $e_{\mathrm{T}}$ and $e_{\mathrm{B}}$ satisfy property (ii) in Lemma 3. Therefore, it is clear that $\left|\mathcal{C}^{\prime}\right|=|\mathcal{C}|-1, a \notin V\left(\mathcal{C}^{\prime}\right), \square(c, v) \notin \mathcal{C}$ for any $v \in V(P)-b$, and $\mathcal{C}^{\prime}$ is a diagonal cover of $P$.

In the main proof, we use operation $\mathcal{C}^{u \rightarrow v}$ for a diagonal rectangle cover $\mathcal{C}$ and $u, v \in$ $V(\mathcal{C})$ by

$$
\mathcal{C}^{u \rightarrow v}:=\mathcal{C} \cup\{\square(v, w) \mid \square(u, w) \in \mathcal{C}\}-\{\square(u, w) \mid w \in V(P)\}
$$

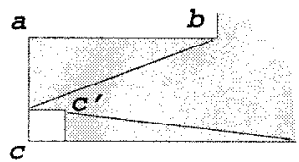

Fig. 11. Proof of Lemma 5. 


\section{Proof of the Theorem}

To simplify the presentation, we do not try to reduce the size $\left\lfloor\frac{3}{2} r\right\rfloor+1$ by one in the case of $r=2,4,6$ until the last several lines of this proof.

Throughout this section we fix $r$ and suppose the following induction hypothesis:

$\left(\mathrm{IH}=\mathrm{IH}_{r}\right)$ For any orthogonal polygon of $r^{\prime}(<r)$ reflex vertices, there is a diagonal rectangle cover of size at most $\left\lfloor\frac{3}{2} r^{\prime}\right\rfloor+1$.

Furthermore, we let $P$ be an orthogonal polygon of $r$ reflex vertices in general integer position which cannot be covered with at most $\left\lfloor\frac{3}{2} r\right\rfloor+1$ diagonal rectangles. Our goal is to prove that such a $P$. does not exist We can assume that $r>0$.

\subsection{A Tab}

We define a $t a b$, which is a special type of neighboring pair which we will not be able to kill easily in our proof. If neighboring edges are connected with a vertical edge and each of them has a reflex end vertex (see Fig. 10(b),(d)), then we call the pair a tab. Especially, if the connecting vertical edge is left or right, the tab is called a left or right $t a b$, respectively. If the length of the top edge is less or greater than the bottom edge, then we call the tab up or down. See Fig. 12.

Lemma 6. Any pair of neighboring edges forms a tab.

Proof. Let a top edge $e_{\mathrm{T}}=a b\left(a<_{\mathrm{H}} b\right)$ and a bottom edge $e_{\mathrm{B}}=c d\left(c<_{\mathrm{H}} d\right)$ be neighboring. Since $r>0$ and $P$ is in general integer position, without loss of generality, we assume that $b<_{\mathrm{H}} d$. Therefore $b$ must be reflex because of Lemma 3(ii). Let $\theta$ be the number of reflex vertices among $a, b, c$, and $d$.

Case $1(\theta=1)$. Because $b<_{\mathrm{H}} d$ and by Lemma 3(ii), the right facing edge of $c d$ is adjacent to $d$ and $\square(\operatorname{right}(a b), c) \subset P$ (see Fig. 10(a)). Consider polygon $P^{\prime}:=$ $P-\square(a, d)$. See Fig. 13. By (IH), $P^{\prime}$ can be covered with at most $\frac{3}{2}(r-1)+1$ diagonal rectangles of $P^{\prime}$. We denote the family of these rectangles by $\mathcal{C}^{\prime}$. We define a new cover by $\mathcal{C}:=\left(\mathcal{C}^{\prime}\right)^{\text {right }(a b) \rightarrow d} \cup\{\square(a, d)\}$. Obviously $\mathcal{C}$ is a diagonal rectangle cover of $P$ the size of which is $\left(\frac{3}{2}(r-1)+1\right)+1=\frac{3}{2} r+\frac{1}{2}$. It contradicts that $P$ is a counterexample.

Case $2(\theta=2)$.

Subcase 2.0 ( $b$ and $d$ are reflex). By Lemma 3(ii), they form a tab.

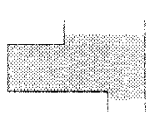

(a)

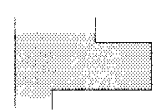

(b)

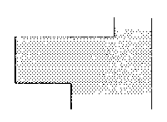

(c)

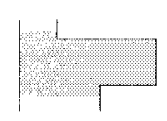

(d)

Fig. 12. (a) A left up tab, (b) a right up tab, (c) a left down tab, and (d) a right down tab. 


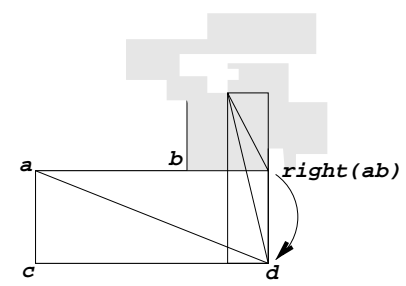

Fig. 13. Case 1.

Subcase 2.1 ( $a$ and $b$ are reflex). By Lemma 3(ii), $c<_{\mathrm{H}} a$. Put $a^{\prime}=\operatorname{left}(a b), b^{\prime}=$ right $(a b)$. Consider region $P-\square\left(c, b^{\prime}\right)$. See Fig. 14(a).

Assume that the resulting region consists of two polygons $P_{i}$ of $r_{i}$ reflex vertices $(i=1,2)$ such that $r_{1}+r_{2}+2=r, a \in P_{1}$, and $b \in P_{2}$. By (IH), $P_{i}$ can be covered with at most $\frac{3}{2} r_{i}+1$ diagonal rectangles $C_{i}$ of $P_{i}$. Then put $\mathcal{C}:=\mathcal{C}_{1}^{a^{\prime} \rightarrow c} \cup \mathcal{C}_{2}^{b^{\prime} \rightarrow d} \cup\{\square(a, d), \square(b, c)\}$. It is clear that all rectangles in $\mathcal{C}$ is a diagonal rectangle of $P$ and $\mathcal{C}$ covers $P$. We see that

$$
|\mathcal{C}|=\left|\mathcal{C}_{1}\right|+\left|\mathcal{C}_{2}\right|+2 \leq \frac{3}{2}\left(r_{1}+r_{2}\right)+2+2=\frac{3}{2}\left(r_{1}+r_{2}+2\right)+1=\frac{3}{2} r+1,
$$

a contradiction.

Subcase 2.2 ( $b$ and c are reflex). Consider region $P-\square(a, d)$. Similarly to the previous subcase, the region can be covered with rectangles $\mathcal{C}^{\prime}=\mathcal{C}_{1} \cup \mathcal{C}_{2}$ of size $\sum_{i=1,2}\left(\frac{3}{2} r_{i}+1\right)$ (for some $\left.r_{i}\left(r=r_{1}+r_{2}+2\right)\right)$. Consider $\mathcal{C}:=\left(\left(\mathcal{C}^{\prime}\right)^{\text {right }(b) \rightarrow d}\right)^{\operatorname{left}(c) \rightarrow a} \cup\{\square(a, d)\}$. It is a diagonal cover of $P$ and its size is

$$
|\mathcal{C}|=\left|\mathcal{C}^{\prime}\right|+1 \leq \frac{3}{2}\left(r_{1}+r_{2}\right)+2+1=\frac{3}{2}\left(r_{1}+r_{2}+2\right)=\frac{3}{2} r,
$$

a contradiction.

Case $3(\theta=3)$. Clearly, it is sufficient to show the case when vertex $d$ is convex. Let $b^{\star}:=\operatorname{right}(b)$. Cut polygon $P$ by $b b^{\star}$, yielding two polygons $P_{i}(i=1,2)$ of $r_{i}$ reflex vertices with $r=r_{1}+r_{2}+1$. Let $P_{1}$ be the polygon containing vertex $a$. By (IH),

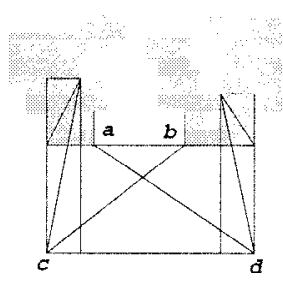

(a)

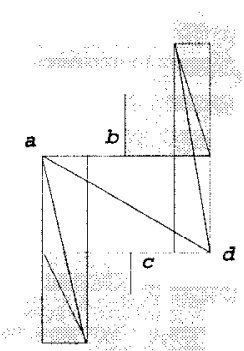

(b)

Fig. 14. (a) Subcase 2.1 and (b) Subcase 2.2. 


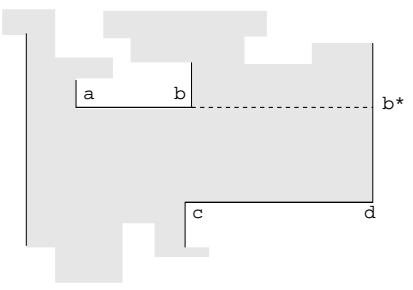

(a)

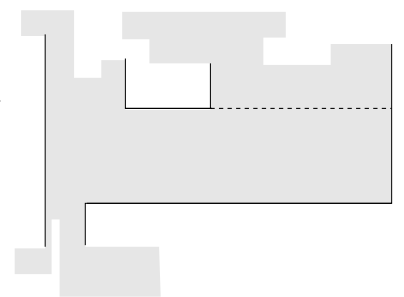

(b)

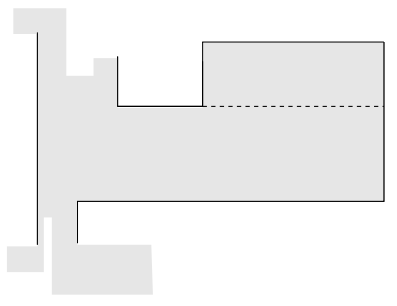

(c)

Fig. 15. (a) Subcase 3.1, (b) Subcase 3.2, and (c) Subsubcase 3.3.1.

polygon $P_{i}(i=1,2)$ is covered with at most $\left\lfloor\frac{3}{2} r_{i}\right\rfloor+1$ diagonal rectangles (of $\left.P_{i}\right)$, say $\mathcal{C}_{i}$. Let $\mathcal{C}:=\mathcal{C}_{1} \cup\left(\mathcal{C}_{2}\right)^{b^{*} \rightarrow d}$. Clearly,

$$
|\mathcal{C}| \leq\left\lfloor\frac{3}{2} r_{1}\right\rfloor+\left\lfloor\frac{3}{2} r_{2}\right\rfloor+2 \leq \frac{3}{2}\left(r_{1}+r_{2}+1\right)+\frac{1}{2}=\frac{3}{2} r+\frac{1}{2}
$$

Subcase $3.1\left(a<_{\mathrm{H}} c\right)$. See Fig. 15. Note that $a b^{\star}$ and $c d$ form a right down tab. By Lemma 4 , we can assume that $b^{\star} \notin V(\mathcal{C})$, which means that all rectangles in $\mathcal{C}$ are diagonal of $P$. Clearly, $\mathcal{C}$ covers $P$ and

$$
|\mathcal{C}| \leq \frac{3}{2} r+\frac{1}{2}
$$

a contradiction.

Subcase $3.2\left(c<_{\mathrm{H}}\right.$ a and $r_{1}$ is odd $)$. Note that $a b^{\star}$ and $c d$ form a right $u p$ tab. If $\square\left(b^{\star}, u\right) \in \mathcal{C}$, then $u=c$. Consider $\mathcal{C}^{+}:=\mathcal{C}^{b^{\star} \rightarrow b} \cup\{\square(a, d)\}$. It covers $P$ and all rectangles in it are diagonal rectangles of $P$. Since $r_{1}$ is odd, its size is

$\left|\mathcal{C}^{+}\right|=|\mathcal{C}|+1 \leq\left\lfloor\frac{3}{2} r_{1}+1\right\rfloor+\left\lfloor\frac{3}{2} r_{2}+1\right\rfloor+1 \leq\left(\frac{3}{2} r_{1}+\frac{1}{2}\right)+\left(\frac{3}{2} r_{2}+1\right)+1=\frac{3}{2} r+1$,

a contradiction.

Subcase $3.3\left(c<_{\mathrm{H}}\right.$ a and $r_{1}$ is even $)$.

Subsubcase 3.3.1 $\left(r_{2}=0\right)$. It is clear that $P=P_{1} \cup \square\left(b^{\prime}, d\right)$ where $b^{\prime}(\neq a)$ is the end vertex of the vertical edge incident to $b$ with $b<_{\mathrm{v}} b^{\prime}$ (i.e., $b^{\prime}=\operatorname{up}(b)$ ). Then $P$ has a diagonal rectangle cover $\mathcal{C}^{+}:=\mathcal{C}_{1} \cup\left\{\square\left(b^{\prime}, d\right)\right\}$. Since $r_{1}=r-1$, its size is

$$
\left|\mathcal{C}^{+}\right|=\left|\mathcal{C}_{1}\right|+1 \leq \frac{3}{2}(r-1)+1+1=\frac{3}{2} r+\frac{1}{2},
$$

a contradiction.

Subsubcase 3.3.2 $\left(r_{2}>0\right)$. Since $r_{1}=r-1-r_{2} \leq r-2$, any orthogonal polygon of $r_{1}+1$ reflex vertices can be covered with the desired number of diagonal rectangles by (IH). Therefore, by Lemma 5, we can assume that $b^{\star} \notin V(\mathcal{C})$ since $r_{1}$ is even and $a b^{\star}$ and $c d$ form a right up tab. It is clear that $\mathcal{C}$ is a diagonal rectangle cover of $P$. As we computed before,

$$
|\mathcal{C}| \leq \frac{3}{2} r+\frac{1}{2}
$$

a contradiction. 


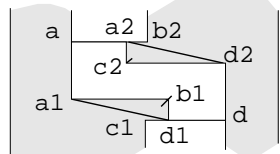

(a)

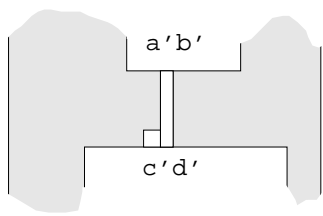

(b)

Fig. 16. (a) Subcase 4.1 and (b) Subcase 4.2 .

Case $4(\theta=4)$. Since $b<_{\mathrm{H}} d$, we have only two subcases.

Subcase $4.1\left(a<_{\mathrm{H}} c\right)$. Let $a_{1}:=\left((a)_{\mathrm{H}},(c)_{\mathrm{V}}+0.1\right), b_{1}:=c+(0.1,0.1), c_{1}:=$ $c, d_{1}=c+(0.1,0), a_{2}:=b+(-0.1,0), b_{2}:=b, c_{2}:=b+(-0.1,-0.1)$, and $d_{2}:=\left((d)_{\mathrm{H}},(b)_{\mathrm{V}}-0.1\right)$. Consider region $P^{\prime}:=P-\left(\square\left(a, c_{2}\right) \cup \square\left(a_{1}, d_{2}\right) \cup \square\left(b_{1}, d\right)\right)$, which consists of two polygons $P_{i}(i=1,2)$ of $r_{i}$ reflex vertices such that $P_{1}$ contains $a_{1}$. See Fig. 16. By (IH), $P^{\prime}$ can be covered with at most $\left\lfloor\frac{3}{2} r_{1}+1\right\rfloor+\left\lfloor\frac{3}{2} r_{2}+1\right\rfloor \leq$ $\left\lfloor\frac{3}{2} r\right\rfloor+2$ diagonal rectangle, say $\mathcal{C}^{\prime}$. By Lemma 4 , we assume that $b_{1}, c_{2} \notin V\left(\mathcal{C}^{\prime}\right)$ and $\square\left(a_{1}, d_{1}\right), \square\left(a_{2}, d_{2}\right) \in \mathcal{C}^{\prime}$. Let $\mathcal{C}:=\left(\left(\mathcal{C}^{\prime}-\square\left(a_{1}, d_{1}\right)-\square\left(a_{2}, d_{2}\right)\right)^{a_{1} \rightarrow a}\right)^{d_{2} \rightarrow d} \cup\{\square(a, d)\}$. It is clear that $\mathcal{C}$ is a diagonal rectangle cover of $P$. The size of it is

$$
|\mathcal{C}|=\left|\mathcal{C}^{\prime}\right|-1 \leq \frac{3}{2} r+1,
$$

a contradiction.

Subcase $4.2\left(c<_{\mathrm{H}} a\right)$. Let $a^{\prime}:=a+\left(\frac{1}{3}, 0\right), b^{\prime}:=a+\left(\frac{2}{3}, 0\right), c^{\prime}:=\left((a)_{\mathrm{H}}+\frac{1}{3},(c)_{\mathrm{V}}\right)$, and $d^{\prime}:=c^{\prime}+\left(\frac{1}{3}, 0\right)$. Consider $P^{\prime}:=P-\square\left(a^{\prime}, d^{\prime}\right)$. Let $P^{\prime}$ consist of two polygons $P_{i}$ $(i=1,2)$ of $r_{i}$ reflex vertices such that $r=r_{1}+r_{2}$ and $P_{1}$ contains vertex $a_{1}$. By (IH), we can cover $P_{i}$ by at most $\left\lfloor\frac{3}{2} r_{i}\right\rfloor+1$ diagonal rectangles, say $\mathcal{C}_{i}$. Let $\mathcal{C}^{\prime}=\mathcal{C}_{1} \cup \mathcal{C}_{2}$. Note that for $u \in\left\{a^{\prime}, b^{\prime}, c^{\prime}, d^{\prime}\right\}$ and $v \in V(P)$, if $\square(u, v) \in \mathcal{C}^{\prime}$, then $v \in\{a, b, c, d\}$.

Subsubcase 4.2.1 ( $r_{1}$ and $r_{2}$ are both odd). This case is easy. By Lemma 4, we can assume that $c^{\prime}, d^{\prime} \notin V\left(\mathcal{C}^{\prime}\right)$ and $\square\left(a^{\prime}, c\right), \square\left(b^{\prime}, d\right) \in \mathcal{C}^{\prime}$. It is clear that $P$ can be covered with $\mathcal{C}:=\mathcal{C}^{\prime} \cup\{\square(a, d), \square(b, c)\}-\left\{\square\left(a^{\prime}, c\right), \square\left(b^{\prime}, d\right)\right\}$, whose members are all diagonal rectangles of $P$, and

$$
|\mathcal{C}|=\left|\mathcal{C}^{\prime}\right| \leq\left(\frac{3}{2} r_{1}-\frac{1}{2}\right)+\left(\frac{3}{2} r_{2}-\frac{1}{2}\right)+2=\frac{3}{2} r+1,
$$

a contradiction.

Subsubcase 4.2.2 (otherwise). Without loss of generality, we can assume that $r_{1}$ is even. By (IH), any orthogonal polygon of $r_{1}+1$ reflex vertices can be covered with at most $\frac{3}{2}\left(r_{1}+1\right)+1$ diagonal rectangles of it since $r_{1} \leq r-2$. Therefore, by Lemmas 4 and 5, we can assume that $a^{\prime}, d^{\prime} \notin V\left(\mathcal{C}^{\prime}\right)$ and $\square\left(a, c^{\prime}\right), \square\left(b^{\prime}, d\right) \in \mathcal{C}^{\prime}$. Consider $\mathcal{C}:=$ $\mathcal{C}^{\prime} \cup\{\square(a, d)\}-\left\{\square\left(a, c^{\prime}\right), \square\left(b^{\prime}, d\right)\right\}$. It is a diagonal rectangle cover of $P$, whose size is

$$
|\mathcal{C}|=\left|\mathcal{C}^{\prime}\right|-1 \leq \frac{3}{2}\left(r_{1}+r_{2}\right)+2-1=\frac{3}{2} r+1,
$$

a contradiction. 


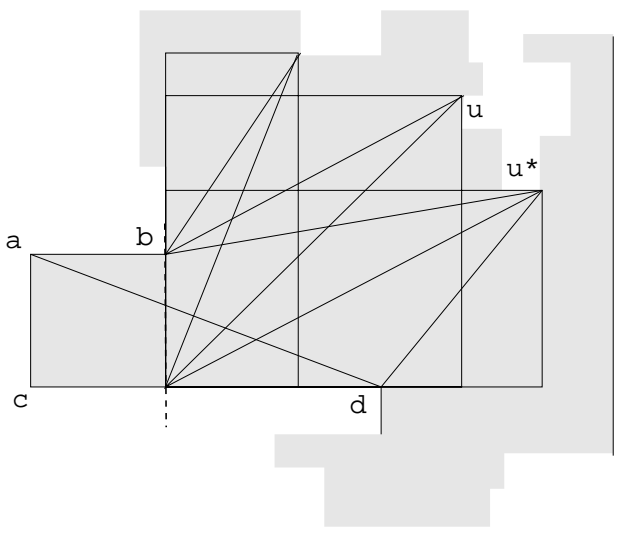

Fig. 17. Proof of Lemma 7.

We also have the following fact.

Lemma 7. The number $r$ of the reflex vertices is odd.

Proof. By Lemmas 3(i) and 6, without loss of generality, we assume that there exists a (left up) tab of top $e_{\mathrm{T}}=a b\left(a<_{\mathrm{H}} b\right)$ and bottom $e_{\mathrm{B}}=c d\left(c<_{\mathrm{H}} d\right)$. Consider $P^{\prime}:=P-\square(c, b)$. See Fig. 17. By (IH), we can cover it with at most $\left\lfloor\frac{3}{2}(r-1)\right\rfloor+1=$ $\left\lfloor\frac{3}{2} r-\frac{1}{2}\right\rfloor$ diagonal rectangles, say $C^{\prime}$. Let $\mathcal{C}:=\left(\mathcal{C}^{\prime}\right)^{\text {down }(b) \rightarrow b} \cup\{\square(a, d)\}$, in which all rectangles are diagonal in $P$. If $\mathcal{C}$ does not cover $P$, then there exists $u \in V(P)$ such that $\square(u$, down $(b)) \in \mathcal{C}^{\prime}, d<_{\mathrm{H}} u$ and $b<_{\mathrm{V}} u$. Consider $u^{\star}$ to be the lowest vertex among such $u$ 's. It is clear that $\mathcal{C} \cup\left\{\square\left(d, u^{\star}\right)\right\}$ is a diagonal rectangle cover of $P$ whose size is at most

$$
|\mathcal{C}|+1=\mathcal{C}^{\prime}+2 \leq\left\lfloor\frac{3}{2} r-\frac{1}{2}\right\rfloor+2=\left\lfloor\frac{3}{2} r+\frac{1}{2}\right\rfloor+1 .
$$

If $r$ is even, then the number is at most $\frac{3}{2} r+1$, a contradiction.

\subsection{Bad Tabs}

We define four types of bad tabs, front or back and simple or chipped bad tabs. See Fig. 18.

First, we define simple bad tabs. We let top edge $e_{\mathrm{T}}=a b$ and bottom edge $e_{\mathrm{B}}=c d$ $\left(a=_{\mathrm{H}} c<_{\mathrm{H}} b<_{\mathrm{H}} d\right)$ form a left up tab with its right facing edge $u v\left(u<_{\mathrm{V}} v\right)$. See Fig. 18(a),(b). Let $b^{\star}(\neq a), b^{\star \star}(\neq b), d^{\star}(\neq c), v^{\star}(\neq u)$ be the vertices adjacent to $b, b^{\star}, d, v$, respectively. If the lowest then rightmost (or leftmost) vertex in region $\left\{x \in P \mid b \leq_{\mathrm{H}} x \leq_{\mathrm{H}} u, b<_{\mathrm{V}} x\right\}$ is $b^{\star}$ (or $v$ ), then we say that the tab forms a left up front (or back) simple bad tab $\left\langle d^{\star}, d, c ; a, b, b^{\star} ; u, v\right\rangle$ with step edge $b^{\star} b^{\star \star}$ (or $v v^{\star}$ ). Note that $u_{6}<_{\mathrm{V}} v_{2}$ if and only if the left up simple bad tab is front. Clearly, $\square(a, \operatorname{right}(d)) \cup\{x \in$ $\left.\mathbf{R}^{2} \mid b \leq_{\mathrm{H}} x \leq_{\mathrm{H}} u,(b)_{\mathrm{V}} \leq(x)_{\mathrm{V}} \leq \min \left\{\left(b^{\star}\right)_{\mathrm{V}},(v)_{\mathrm{V}}\right\}\right\} \subset P$. 


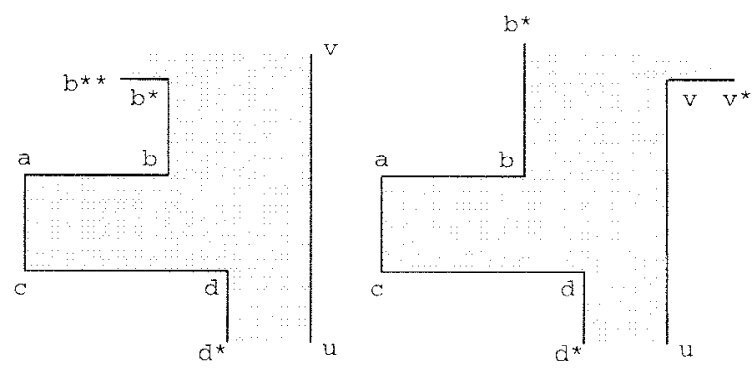

(a)

(b)

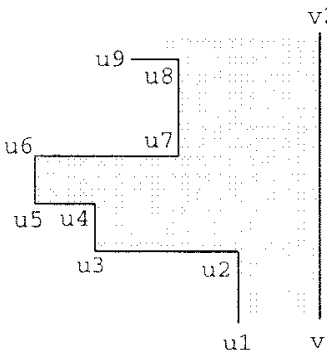

(c)

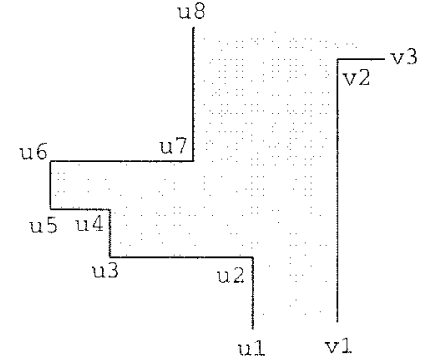

(d)

Fig. 18. (a) A left up front simple bad tab, (b) a left up back simple bad tab, (c) a left up facing chipped bad tab, and (d) a left up back chipped bad tab.

Second, we define chipped bad tabs. Let $u_{1}, \ldots, u_{9}, v_{1}, v_{2}, v_{3}$ be distinct vertices such that $u_{i} u_{i+1} \in E(P)(i=1, \ldots, 8)$ and $v_{j} v_{j+1} \in E(P)(j=1,2)$. Assume that

(i) $u_{6} u_{7}, u_{5} u_{4}\left(u_{5}=_{\mathrm{H}} u_{6}<_{\mathrm{H}} u_{7}<_{\mathrm{H}} u_{4}\right)$ are top and bottom edges, respectively, which form a left "down" tab with right facing edge $v_{1} v_{2}\left(v_{1}<_{\mathrm{V}} v_{2}\right)$,

(ii) the highest then leftmost and rightmost vertices in region $\left\{x \in P \mid u_{4} \leq_{\mathrm{H}} x \leq_{\mathrm{H}}\right.$ $\left.v_{1}, x<_{\mathrm{V}} u_{4}\right\}$ are $u_{3}$ and $u_{2}$, respectively, and finally

(iii) $u_{7}<_{\mathrm{H}} u_{2}$.

If the lowest then rightmost (or leftmost) vertex in region $\left\{x \in P \mid u_{7} \leq_{\mathrm{H}} x \leq_{\mathrm{H}} v_{1}, u_{7}<\mathrm{V}\right.$ $x\}$ is $u_{8}$ (or $v_{2}$ ), then we say that the tab $\left\langle u_{6} u_{7}, u_{5} u_{4}\right\rangle$ forms a left up front (or back) chipped bad tab $\left\langle u_{1}, u_{2}, u_{3} ; u_{6}, u_{7}, u_{8} ; v_{1}, v_{2}\right\rangle$ with chip edge $u_{5} u_{4}$ and with step edge $u_{8} u_{9}$ (or $\left.v_{2} v_{3}\right)$. Clearly, $v_{1}<_{\mathrm{V}} u_{2}$. Note that $\square\left(u_{3}\right.$, right $\left.\left(u_{4}\right)\right) \cup \square\left(u_{5}\right.$, right $\left.\left(u_{6}\right)\right) \cup\{x \in$ $\left.\mathbf{R}^{2} \mid u_{7} \leq_{\mathrm{H}} x \leq_{\mathrm{H}} v_{1},\left(u_{7}\right)_{\mathrm{V}} \leq(x)_{\mathrm{V}} \leq \min \left\{\left(u_{8}\right)_{\mathrm{V}},\left(v_{2}\right)_{\mathrm{V}}\right\}\right\} \subset P$. See Fig. 18(c), (d). We define bad tabs for right or down tabs similarly. See Figs. 19-21.

Lemma 8. Any tab forms a bad tab.

Proof. Without loss of generality, we can assume that the tab is a left up tab. Let top $e_{\mathrm{T}}=a b$ and bottom $e_{\mathrm{T}}=c d\left(a==_{\mathrm{H}} c<_{\mathrm{H}} b<_{\mathrm{H}} d\right)$ form a left up tab with facing edge $v_{1} v_{2}\left(v_{1}<_{\mathrm{V}} v_{2}\right)$. Let $b^{\prime}(\neq a), d^{\prime}(\neq c)$ be vertices adjacent to $b, d$, respectively, and 


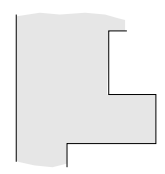

(a)

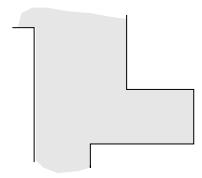

(b)

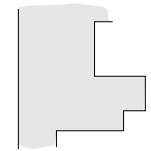

(c)

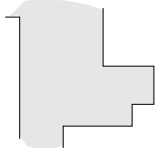

(d)

Fig. 19. (a) A right up front simple bad tab, (b) a right up back simple bad tab, (c) a right up facing chipped bad tab, and (d) a right up back chipped bad tab.

let vertex $w_{\mathrm{L}}$ or $w_{\mathrm{R}}$ be the lowest then leftmost or rightmost vertex within region $\{x \in$ $\left.P \mid b \leq_{\mathrm{H}} x \leq_{\mathrm{H}} v_{1}, b<_{\mathrm{V}} x\right\}$, respectively. Since the region contains $b^{\prime}$ and $v_{2}$, vertices $w_{\mathrm{L}}$ and $w_{\mathrm{R}}$ must exist. Note that $w_{\mathrm{L}}=_{\mathrm{V}} w_{\mathrm{R}}$. They may be possibly equal to one another.

Case $0\left(w_{\mathrm{L}}=w_{\mathrm{R}}\right)$. Since any horizontal edge contains two distinct vertices, if $w_{\mathrm{L}}=w_{\mathrm{R}}$, then $w_{\mathrm{L}}=w_{\mathrm{R}} \in\left\{b^{\prime}, v_{2}\right\}$ and the vertex $w_{\mathrm{L}}=w_{\mathrm{R}}$ must be reflex. Therefore the tab forms a front or back simple bad tab.

Case $1\left(b<_{\mathrm{H}} w_{\mathrm{R}}<_{\mathrm{H}} d\right)$. See Fig. 22(a). We see that $P^{\prime}:=P-\square(b, c)$ is covered with at most $\frac{3}{2}(r-1)+1=\frac{3}{2} r-\frac{1}{2}$ diagonal rectangles, say $\mathcal{C}^{\prime}$. Note that if $\square(\operatorname{down}(b), x) \in \mathcal{C}^{\prime}$, then $x \leq_{\mathrm{H}} w_{\mathrm{R}}<_{\mathrm{H}} d$. Then $\mathcal{C}:=\left(\mathcal{C}^{\prime}\right)^{\text {down }(b) \rightarrow b} \cup\{\square(a, d)\}$ is a diagonal rectangle cover of $P$ whose size is at most $\left|\mathcal{C}^{\prime}\right|+1 \leq \frac{3}{2} r+\frac{1}{2}$.

Case $2\left(d<_{\mathrm{H}} w_{\mathrm{R}} \leq_{\mathrm{H}} v_{2}\right.$ and $\left.w_{\mathrm{L}} \neq b^{\prime}\right)$. See Fig. 22(b). Put $w_{\mathrm{L}}^{\prime}:=w_{\mathrm{L}}+(0,0.5)$ and $b^{\star}:=\operatorname{left}\left(w_{\mathrm{L}}\right)+(0,0.5)$. Consider region $P^{\prime}:=P-\square\left(b^{\star}, w_{\mathrm{L}}\right)$. We see that $P^{\prime}$ is covered with at most $\frac{3}{2}\left(r_{1}+r_{2}\right)+2=\frac{3}{2} r+\frac{1}{2}$ diagonal rectangles, say $\mathcal{C}^{\prime}$ (for some $\left.r_{i}\left(r_{1}+r_{2}+1=r\right)\right)$. If $\square\left(\operatorname{left}\left(w_{\mathrm{L}}\right), x\right) \in \mathcal{C}^{\prime}$, then $x=d$. Because of Lemma 4 and $\square(a, d) \cup \square\left(b, w_{\mathrm{R}}\right) \supset \square\left(\operatorname{left}\left(w_{\mathrm{L}}\right), d\right)$, we can assume that $\square(a, d) \in \mathcal{C}^{\prime} \not \supset$ $\square\left(\operatorname{left}\left(w_{\mathrm{L}}\right), d\right)$. Consider $\mathcal{C}:=\left(\left(\mathcal{C}^{\prime}\right)^{b^{*} \rightarrow b}\right)^{w_{\mathrm{L}}^{\prime} \rightarrow w_{\mathrm{L}}}$. It is clear that $\mathcal{C}$ is the desired diagonal rectangle cover of $P$.

Case $3\left(w_{\mathrm{R}}=v_{2}\right.$ and $\left.w_{\mathrm{L}}=b^{\prime}\right)$. See Fig. 22(c). It is seen that polygon $P^{\prime}:=P-$ $\square(b, c)-\square\left(b^{\prime}, d\right)-\square\left(v_{2}, d\right)$ can be covered with at most $\frac{3}{2}(r-2)+1$ diagonal rectangles, say $\mathcal{C}^{\prime}$. Consider $\mathcal{C}:=\left(\mathcal{C}^{\prime}\right)^{\operatorname{right}(d) \rightarrow v_{2}} \cup\left\{\square(a, d), \square\left(b, v_{2}\right), \square\left(d, v_{2}\right)\right\}$, which is the desired diagonal rectangle cover of $P$.

Case $4\left(d<_{\mathrm{H}} w_{\mathrm{R}}<_{\mathrm{H}} v_{2}\right.$ and $\left.w_{\mathrm{L}}=b^{\prime}\right)$. We show that the tab forms a left down chipped bad tab. Let vertex $z_{\mathrm{L}}, z_{\mathrm{R}}$ be the highest then leftmost or rightmost vertex within region $\left\{x \in P \mid d \leq_{\mathrm{H}} x \leq_{\mathrm{H}} v_{1}, b>_{\mathrm{V}} x\right\}$, respectively.

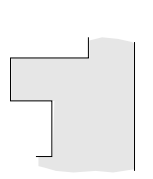

(a)

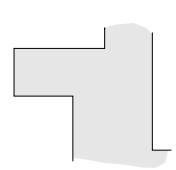

(b)

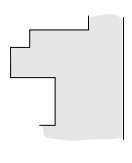

(c)

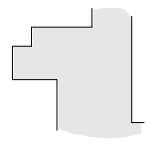

(d)

Fig. 20. (a) A left down front simple bad tab, (b) a left down back simple bad tab, (c) a left down facing chipped bad tab, and (d) a left down back chipped bad tab. 


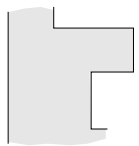

(a)

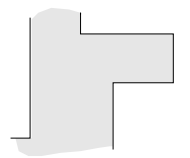

(b)

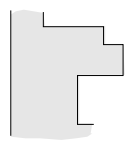

(c)

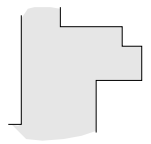

(d)

Fig. 21. (a) A right down front simple bad tab, (b) a right down back simple bad tab, (c) a right down facing chipped bad tab, and (d) a right down back chipped bad tab.

Subcase $4.0\left(z_{\mathrm{L}}=z_{\mathrm{R}}\right)$. If $z_{\mathrm{L}}=z_{\mathrm{R}}$, then $z_{\mathrm{L}}=z_{\mathrm{R}} \in\left\{d^{\prime}, v_{1}\right\}$ and it must be reflex. Therefore the tab forms a (left down) front or back chipped bad tab.

Subcase $4.1\left(d<_{\mathrm{H}} \quad z_{\mathrm{R}}<_{\mathrm{H}} \quad w_{\mathrm{R}}\right)$. See Fig. 23(a). Consider $P^{\prime}:=P-\square\left(b^{\prime}, d\right)-$ $\square(b, c)$. It can be covered with at most $\frac{3}{2}(r-2)+1$ diagonal rectangles, say $\mathcal{C}^{\prime}$. Since $\square(\operatorname{up}(d), x) \in \mathcal{C}^{\prime}$ implies $x<_{\mathrm{H}} w_{\mathrm{R}}$, it is clear that $P$ is covered with $\mathcal{C}:=\left(\mathcal{C}^{\prime}\right)^{\operatorname{up}(d) \rightarrow d} \cup$ $\left\{\square(a, d), \square\left(b^{\prime}, d\right), \square\left(w_{\mathrm{R}}, d\right)\right\}$, which is the desired diagonal rectangle cover of $P$ whose size is at most $\frac{3}{2} r+1$.

Subcase $4.2\left(w_{\mathrm{R}}<_{\mathrm{H}} z_{\mathrm{R}} \leq_{\mathrm{H}} \quad v_{1}\right.$ and $\left.z_{\mathrm{L}}=d^{\prime}\right)$. See Fig. 23(b). Consider $P^{\prime}:=$ $P-\square\left(b^{\prime}, d\right)-\square(b, c)$. It can be covered with at most $\frac{3}{2}(r-2)+1$ diagonal rectangles, say $\mathcal{C}^{\prime}$. If $\square(\operatorname{up}(d), x) \in \mathcal{C}^{\prime}$, then we can assume that $x=z_{\mathrm{R}}$. By Lemma 4, we can also assume that $\square\left(z_{\mathrm{R}}, \operatorname{up}(d)\right) \in \mathcal{C}^{\prime}$. We easily see that $\mathcal{C}:=\mathcal{C}^{\prime} \cup\{\square(a, d)$, $\left.\square\left(b^{\prime}, d\right), \square\left(w_{\mathrm{R}}, d^{\prime}\right), \square\left(z_{\mathrm{R}}, w_{\mathrm{R}}\right)\right\}-\square\left(z_{\mathrm{R}}\right.$, up $\left.(d)\right)$ is the desired diagonal rectangle cover of $P$.

Subcase $4.3\left(w_{\mathrm{R}}<_{\mathrm{H}} z_{\mathrm{R}} \leq_{\mathrm{H}} v_{1}\right.$ and $\left.z_{\mathrm{L}} \neq d^{\prime}\right)$. See Fig. 23(c). Let $d^{-}:=\operatorname{left}\left(z_{\mathrm{L}}\right), d^{--}:=$ $d^{-}+(0,-0.5), z^{-}:=z_{\mathrm{L}}+(0,-0.5)$, and $P^{\prime}:=P-\square\left(z_{\mathrm{L}}, d^{--}\right)-\square(a, d)-\square\left(b^{\prime}, d\right)$ consist of two polygons $P_{i}(i=1,2)$ of $r_{i}$ reflex vertices such that $d \in P_{1}$. By (IH), $P^{\prime}$ can be covered with at most $\left\lfloor\frac{3}{2} r_{1}+1\right\rfloor+\left\lfloor\frac{3}{2} r_{2}+1\right\rfloor \leq \frac{3}{2}(r-3)+2=\frac{3}{2} r-2.5$ diagonal rectangles, say $\mathcal{C}^{\prime}$. Obviously $\square\left(d^{-}, x\right) \in \mathcal{C}^{\prime}$ implies $x=w_{\mathrm{R}}$.

Subsubcase 4.3 .1 ( $r_{1}$ is even). By Lemma 5, we assume that $\operatorname{up}(d) \notin V\left(\mathcal{C}^{\prime}\right)$ and $\square\left(w_{\mathrm{R}}, d^{-}\right) \in \mathcal{C}^{\prime}$. Consider $\mathcal{C}:=\left(\left(\mathcal{C}^{\prime}\right)^{z^{-} \rightarrow z_{\mathrm{L}}}\right)^{d^{--} \rightarrow d} \cup\left\{\square(a, d), \square\left(b, w_{\mathrm{R}}\right), \square\left(w_{\mathrm{R}}, d\right)\right.$, $\left.\square\left(d, z_{\mathrm{R}}\right)\right\}-\square\left(d^{-}, w_{\mathrm{R}}\right)$, which is a diagonal rectangle cover of $P$ whose size is at most $\left|\mathcal{C}^{\prime}\right|+3 \leq \frac{3}{2} r+\frac{1}{2}$, a contradiction.

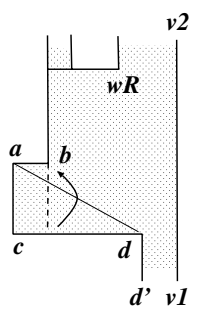

(a)

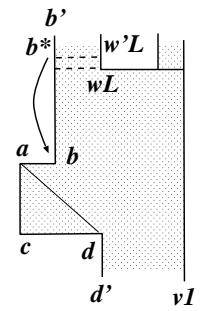

(b)

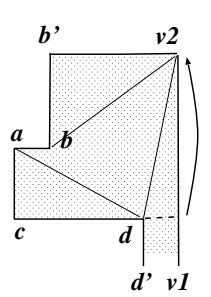

(c)

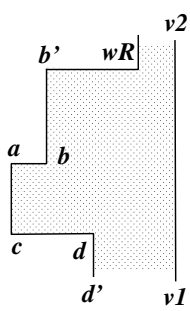

(d)

Fig. 22. (a) Case 1, (b) Case 2, (c) Case 3, and (d) Case 4. 


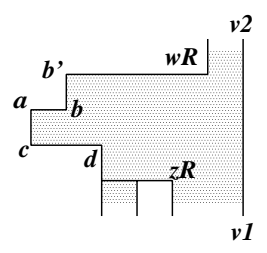

(a)

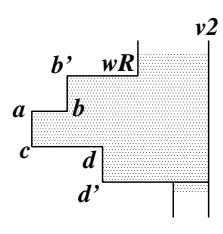

(b)

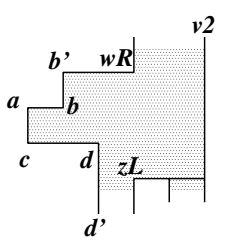

(c)

Fig. 23. (a) Subcase 4.1, (b) Subcase 4.2, and (c) Subcase 4.3.

Subsubcase 4.3.2 ( $r_{1}$ is odd). By Lemma 4, we can assume that $d^{-} \notin V\left(\mathcal{C}^{\prime}\right)$ and $\square\left(\operatorname{up}(d), z_{\mathrm{R}}\right) \in \mathcal{C}^{\prime}$. Consider $\mathcal{C}:=\left(\left(\mathcal{C}^{\prime}\right)^{z^{-} \rightarrow z_{\mathrm{L}}}\right)^{d^{--} \rightarrow d} \cup\left\{\square(a, d), \square\left(b, w_{\mathrm{R}}\right), \square\left(w_{\mathrm{R}}, d\right)\right.$, $\left.\square\left(d, z_{\mathrm{R}}\right), \square\left(w_{\mathrm{R}}, z_{\mathrm{R}}\right)\right\}-\square\left(\mathrm{up}(d), z_{\mathrm{R}}\right)$. It is a diagonal rectangle cover of $P$. Note that $r_{2}$ is also odd because $r_{2}=r-r_{1}-3$ and $r$ is odd by Lemma 7. The size of it is at most $\left|\mathcal{C}^{\prime}\right|+4 \leq\left(\frac{3}{2} r_{1}+\frac{1}{2}\right)+\left(\frac{3}{2} r_{2}+\frac{1}{2}\right)+4=\frac{3}{2}\left(r_{1}+r_{2}+3\right)+\frac{1}{2}=\frac{3}{2} r+\frac{1}{2}$, a contradiction.

\subsection{Bad Tab Pairs}

We define two types of bad tab pairs. Let $\left\langle u_{1}, u_{2}, u_{3} ; u_{4}, u_{5}, u_{6} ; v_{1}, v_{2}\right\rangle$ be a left up back chipped bad tab with step edge $v_{2} v_{3}$ and chip edge $u_{4}^{\prime} u_{5}^{\prime}$. If there are distinct vertices $v_{3}, \ldots, v_{6}$ such that $\left\langle v_{6}, v_{5}, v_{4} ; v_{3}, v_{2}, v_{1} ; u_{6}, u_{5}\right\rangle$ is a right down simple (or chipped) bad tab with step edge $u_{4} u_{5}$, then we call $\left\langle u_{1}, \ldots, u_{6} ; v_{1}, \ldots, v_{6}\right\rangle$ a left up simple (or chipped) bad tab pair. See Fig. 24. We define right or down bad pairs, similarly. A right down chipped bad tab pair is the same as a left up chipped bad tab pair.

\section{Lemma 9. Polygon P contains a bad tab pair.}

Proof. By Lemmas 3(i), 6, and 8, we can assume that $P$ contains an "up" simple or chipped bad tab. We take the highest one, say $T_{0}$, among up bad tabs. We assume that $T_{0}=\left\langle u_{1}, u_{2}, u_{3} ; u_{4}, u_{5}, u_{6} ; u_{11}, u_{12}\right\rangle$ is a left up bad tab. Bad tab $T_{0}$ has a step edge $e^{s 0}$ which is a bottom edge. Bottom edge $e=e^{s 0}$ has its nearest visible edge nv $(e)$, which also has its nearest visible nv $(\operatorname{nv}(e))$. Because the number of edges is finite, there
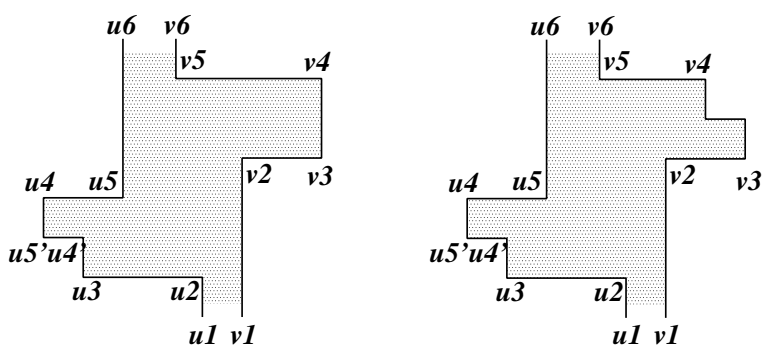

Fig. 24. A simple and a chipped bad tab pair. 

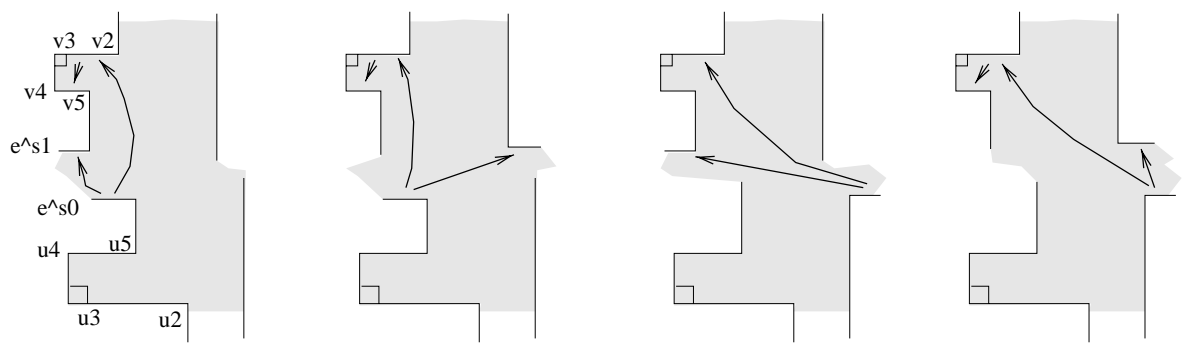

Fig. 25. $T_{0}$ and $T_{1}$ must overlap.

exists an integer $i$ such that $\mathrm{nv}^{i-1}(e)=\mathrm{nv}^{i+1}(e)$, and a pair of $\mathrm{nv}^{i-1}(e)$ and $\mathrm{nv}^{i}(e)$ is a tab $T(e)$. Then the tab $T(e)$ forms a bad tab $T_{1}=\left\langle v_{1}, v_{2}, v_{3} ; v_{4}, v_{5}, v_{6} ; v_{11}, v_{12}\right\rangle$ by Lemma 8 . Since clearly $T_{1}$ is strictly higher than $T_{0}$, by the way of choosing $T_{0}, T_{1}$ must be a "down" bad tab.

Suppose that $e=e^{s 0}$ is not equal to the bottom edge $v_{4} v_{5}$ of the tab forming $T_{1}$. See Fig. 25. In this case, by observing the shapes of bad tabs, whether they are simple or chipped, the step (bottom) edge $e^{s 0}$ of $T_{0}$ must be lower than the step (top) edge $e^{s 1}$ of $T_{1}$. If top $e^{s 1}$ does not see bottom $e^{s 0}$, then there exists another top edge $e_{\mathrm{T}}^{\prime}$ lower than $e^{s 1}$ which sees $e^{s 0}$. Anyway there exists a top edge $e_{\mathrm{T}}$ such that $e_{\mathrm{T}}$ sees $e^{s 0}$ and $e^{s 0}<_{\mathrm{V}} e_{\mathrm{T}}<_{\mathrm{V}} v_{4} v_{5}$ where $v_{4} v_{5}$ is the bottom edge of the tab forming $T_{1}$. Edge $e_{\mathrm{T}}$ may possibly equal $e^{s 1}$. Since $e_{\mathrm{T}}$ sees $e^{s 0}$, for every $i, \mathrm{nv}^{i}\left(e^{s 0}\right) \leq_{\mathrm{V}} e_{\mathrm{T}}$. Then $v_{4} v_{5} \leq_{\mathrm{v}} e_{\mathrm{T}}<\mathrm{V} v_{4} v_{5}$, a contradiction.

Thus $e=e^{s 0}$ equals the bottom edge $v_{4} u_{5}$ of the tab forming down bad tab $T_{1}$. It implies that the top edge $u_{4} u_{5}$ of the tab forming $T_{0}$ is equal to the step (top) edge of down bad tab $T_{1}$.

Case 1 (one bad tab is front and the other is back). This is impossible because of the shapes of bad tabs.

Case 2 (both bad tabs $T_{0}, T_{1}$ are front). See Fig. 26(a),(b). Edge $v_{4} v_{5}$ is the step edge of $T_{0}$ and $u_{11}=v_{12}$ and $u_{12}=v_{11}$. Without loss of generality, we can assume that $v_{2}<_{\mathrm{H}} u_{2}$. Consider $P^{\prime}:=P-\square\left(u_{4}, \operatorname{down}\left(u_{5}\right)\right)$. Note that if $\square\left(\operatorname{down}\left(u_{5}\right), x\right)$ is a diagonal rectangle of $P^{\prime}$, then $x=v_{2}$.

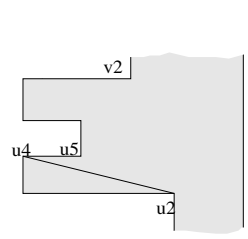

(a)

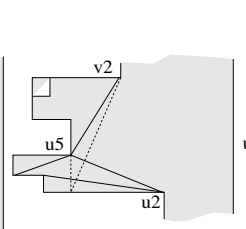

(b)

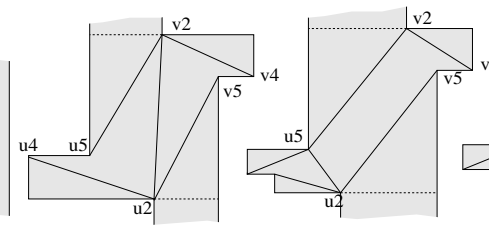

(c) (d)

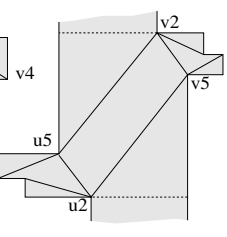

(e)

Fig. 26. (a) Subcase 2.1, (b) Subcase 2.2, (c) Subcase 3.1, (d) Subcase 3.2, and (e) Subcase 3.3. 
Subcase $2.1\left(T_{0}\right.$ is simple $)$. By $(\mathrm{IH}), P^{\prime}$ can be covered with at most $\frac{3}{2}(r-1)+1$ diagonal rectangles, say $\mathcal{C}^{\prime}$. Consider

$$
\mathcal{C}:=\left(\mathcal{C}^{\prime}\right)^{\operatorname{down}\left(u_{5}\right) \rightarrow u_{5}} \cup\left\{\square\left(u_{2}, u_{4}\right)\right\} .
$$

It is a diagonal rectangle cover of $P$ and the size is at most $|\mathcal{C}| \leq\left|\mathcal{C}^{\prime}\right|+1 \leq \frac{3}{2} r+\frac{1}{2}$, a contradiction.

Subcase 2.2 ( $T_{0}$ is chipped). By (IH), $P^{\prime}$ can be covered with at most $\frac{3}{2}(r-2)+1$ diagonal rectangles, say $\mathcal{C}^{\prime}$. Let the chipped edge of $T_{0}$ be $u_{5}^{\prime} u_{4}^{\prime}\left(u_{5}^{\prime}<_{\mathrm{H}} u_{4}^{\prime}\right)$. Consider

$$
\mathcal{C}:=\left(\mathcal{C}^{\prime}\right)^{\operatorname{down}\left(u_{5}\right) \rightarrow u_{5}} \cup\left\{\square\left(u_{5}^{\prime}, u_{5}\right), \square\left(u_{4}^{\prime}, u_{2}\right), \square\left(u_{5}, u_{2}\right)\right\} .
$$

Clearly, $\mathcal{C}$ is a diagonal rectangle cover of $P$ whose size is at most $\left|\mathcal{C}^{\prime}\right|+3 \leq \frac{3}{2} r+1$, a contradiction.

Case 3 (both bad tabs $T_{0}, T_{1}$ are back).

Subcase 3.1 (both bad tabs are simple). See Fig. 26(c). By (IH), $P^{\prime}:=P-\square\left(\operatorname{right}\left(u_{2}\right)\right.$, left $\left.\left(v_{2}\right)\right)-\square\left(u_{2}, u_{4}\right)-\square\left(v_{2}, v_{4}\right)$ can be covered with at most $\frac{3}{2}(r-4)+2=\frac{3}{2} r-4$ diagonal rectangles, say $\mathcal{C}^{\prime}$. Put

$\mathcal{C}:=\left(\left(\mathcal{C}^{\prime}\right)^{\operatorname{right}\left(u_{2}\right) \rightarrow v_{5}}\right)^{\operatorname{left}\left(v_{2}\right) \rightarrow u_{5}} \cup\left\{\square\left(u_{2}, u_{4}\right), \square\left(u_{2}, v_{2}\right), \square\left(u_{2}, v_{5}\right), \square\left(u_{5}, v_{2}\right), \square\left(v_{2}, v_{4}\right)\right\}$.

Obviously $\mathcal{C}$ is a diagonal rectangle cover of $P$ such that $|\mathcal{C}|=\left|\mathcal{C}^{\prime}\right|+5 \leq \frac{3}{2} r+1$, a contradiction.

Subcase 3.2 ( $T_{0}$ is chipped and $T_{1}$ is simple). See Fig. 26(d). We can assume that $u_{2}<_{\mathrm{H}} v_{2}$. Otherwise, they form a simple bad tab pair. Let $u_{5}^{\prime} u_{4}^{\prime}\left(u_{5}^{\prime}<_{\mathrm{H}} u_{4}^{\prime}\right)$ denote the chip edge of $T_{0}$. By $(\mathrm{IH}), P^{\prime}:=P-\square\left(\operatorname{right}\left(u_{2}\right), \operatorname{left}\left(v_{2}\right)\right)-\square\left(u_{2}, u_{4}^{\prime}\right)-\square\left(u_{5}^{\prime}, u_{5}\right)-\square\left(v_{2}, v_{4}\right)$ can be covered with at most $\frac{3}{2}(r-5)+2=\frac{3}{2} r-5.5$ diagonal rectangles, say $\mathcal{C}^{\prime}$. Put

$$
\begin{aligned}
\mathcal{C}:= & \left(\left(\mathcal{C}^{\prime}\right)^{\operatorname{right}\left(u_{2}\right) \rightarrow v_{5}}\right)^{\operatorname{left}\left(v_{2}\right) \rightarrow u_{5}} \\
& \cup\left\{\square\left(u_{2}, u_{4}^{\prime}\right), \square\left(u_{5}^{\prime}, u_{5}\right), \square\left(u_{2}, u_{5}\right), \square\left(u_{2}, v_{5}\right), \square\left(u_{5}, v_{2}\right), \square\left(v_{2}, v_{4}\right)\right\} .
\end{aligned}
$$

Clearly, $\mathcal{C}$ is a diagonal rectangle cover of $P$ such that $|\mathcal{C}|=\left|\mathcal{C}^{\prime}\right|+6 \leq \frac{3}{2} r+\frac{1}{2}$, a contradiction.

Subcase 3.3 (both are chipped). See Fig. 26(e). We can assume that $u_{2}<_{\mathrm{H}} v_{2}$. Otherwise, they form a chipped bad tab pair. Let $u_{5}^{\prime} u_{4}^{\prime}\left(u_{5}^{\prime}<_{\mathrm{H}} u_{4}^{\prime}\right)$ and $v_{4}^{\prime} v_{5}^{\prime}\left(v_{4}^{\prime}<_{\mathrm{H}} v_{5}^{\prime}\right)$ denote the chip edges of $T_{0}$ and $T_{1}$, respectively. By (IH), $P^{\prime}:=P-\square\left(\operatorname{right}\left(u_{2}\right), \operatorname{left}\left(v_{2}\right)\right)-\square\left(u_{2}, u_{4}^{\prime}\right)-$ $\square\left(u_{5}^{\prime}, u_{5}\right)-\square\left(v_{2}, v_{4}^{\prime}\right)-\square\left(v_{5}^{\prime}, v_{5}\right)$ can be covered with at most $\frac{3}{2}(r-6)+2=\frac{3}{2} r-7$ diagonal rectangles, say $\mathcal{C}^{\prime}$. Put

$$
\begin{aligned}
\mathcal{C}:= & \left(\left(\mathcal{C}^{\prime}\right)^{\operatorname{right}\left(u_{2}\right) \rightarrow v_{5}}\right)^{\operatorname{left}\left(v_{2}\right) \rightarrow u_{5}} \\
& \cup\left\{\square\left(u_{2}, u_{4}^{\prime}\right), \square\left(u_{5}^{\prime}, u_{5}\right), \square\left(u_{2}, u_{5}\right), \square\left(u_{2}, v_{5}\right), \square\left(u_{5}, v_{2}\right), \square\left(v_{2}, v_{4}^{\prime}\right),\right. \\
& \left.\square\left(v_{5}^{\prime}, v_{5}\right), \square\left(v_{2}, v_{5}\right)\right\} .
\end{aligned}
$$



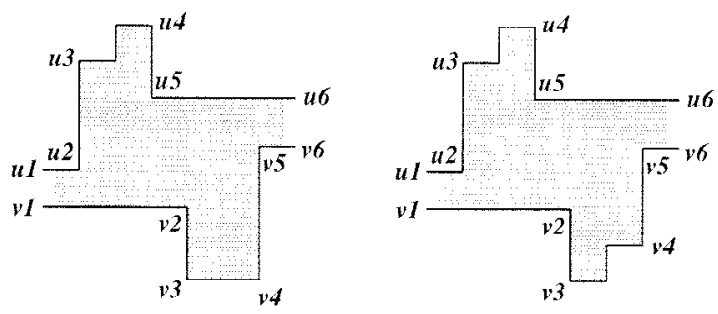

Fig. 27. Bad tab pairs rotated $90^{\circ}$ clockwise.

Clearly, $\mathcal{C}$ is a diagonal rectangle cover of $P$ such that $|\mathcal{C}|=\left|\mathcal{C}^{\prime}\right|+8 \leq \frac{3}{2} r+1$, a contradiction.

We finish the proof of the main theorem.

Proof of Theorem 1'. By Lemma 9, we can assume a left up simple or chipped bad tab pair $\left\langle u_{1}, \ldots, u_{6} ; v_{1}, \ldots, v_{6}\right\rangle$. Until now, we have discussed "horizontal tabs" each of which consists of a neighboring pair of horizontal edges. From now on, we discuss “vertical tabs." Equivalently, rotate our polygon $90^{\circ}$ clockwise. See Fig. 27.

For example, $u_{1} u_{2}, u_{5} u_{6}$ become a top horizontal edge. Note that top edge $u_{1} u_{2}$ sees bottom edge $v_{1} v_{2}$. Then there exists a tab between them. By Lemma 8 , it must be a bad tab, say $T$. Clearly, $T$ must be a left type because of vertical edge $v_{4} v_{5}$.

We observe that the step edge of $T$ must be equal to $v_{5} v_{6}$, which means $T$ should be an up type. Therefore $u_{1} u_{2}$ is the top edge of the tab forming $T$. Furthermore, $v_{1} v_{2}$ is the bottom edge of the tab forming simple bad tab $T$ or the lowest bottom edge among bottom edges in chipped bad tab $T$. Thus $\left\langle v_{3}, v_{2}, v_{1} ; u_{1}, u_{2}, u_{3} ; v_{4}, v_{5}\right\rangle$ is a left up bad tab.

Similarly we can discuss edges $u_{5} u_{6}$ and $v_{5} v_{6}$ instead of $u_{1} u_{2}$ and $v_{1} v_{2}$ then $\left\langle u_{4}, u_{5}, u_{6}\right.$; $\left.v_{6}, v_{5}, v_{4} ; u_{3}, u_{2}\right\rangle$ is a right down bad tab. Consequently we have only four cases with at most eight reflex vertices. It is easily checked that all of the cases can be covered with the desired number of diagonal rectangles.

Therefore we have proven that any orthogonal polygon of $r$ reflex vertices can be covered with at most $\left\lfloor\frac{3}{2} r\right\rfloor+1$ diagonal rectangles.

Sketch proof for $r=2,4,6$ : We show that the covering size can be reduced by one when $r=2,4,6$. It is easily checked for $r=2,4$. Consider $r=6$. Since any orthogonal polygon of two or four vertices can be covered with three or six diagonal rectangles respectively, we can see that there exists a bad pair by repeating the same

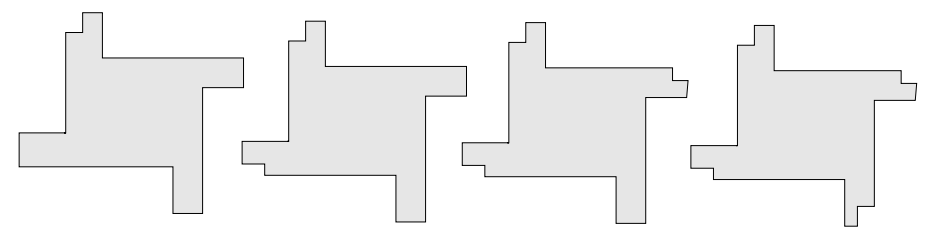

Fig. 28. The easy final cases. 


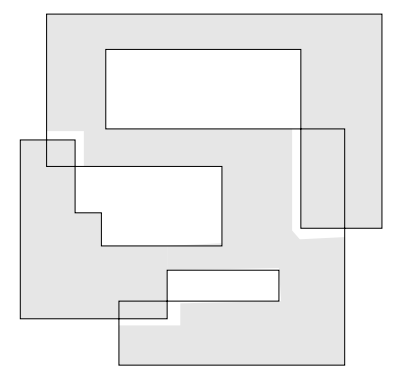

Fig. 29. A self-overlapping orthogonal polygon.

arguments of Lemmas 6, 8, and 9 (though unfortunately we cannot apply the argument of Lemma 7). See Fig. 24. Since the bad pair has five or six of the six reflex vertices, only five cases remain. It is quite easy to show that there is a cover of size at most nine in any of the cases. The theorem has been shown.

\section{Remarks}

We introduced Lemma 2 as a basic property with a short proof in Section 3, and used it in the proofs of Proposition 1 and Theorem 1 in order to assume that $P$ is in general integer position for convenience. However, Lemma 2 is not necessary for either proofs. The argument of Proposition 1 works even if $P$ is not in general integer position. Also it is easily seen that in the proof of the theorem if $P$ is not in general integer position and $u, v$ are two nonadjacent vertices with the same horizontal or vertical coordinate, then the induction hypothesis on $r$ yields the theorem when we cut $P$ into two polygons by vertical or horizontal line $u v$.

Note that our proofs work for more general orthogonal polygons. For example, the theorem holds for "self-overlapping" orthogonal polygons such as that shown in Fig. 29.

It is natural to ask about the relationship between the covering number and the independence number for this diagonal rectangle cover problem as Chvátal asked for the

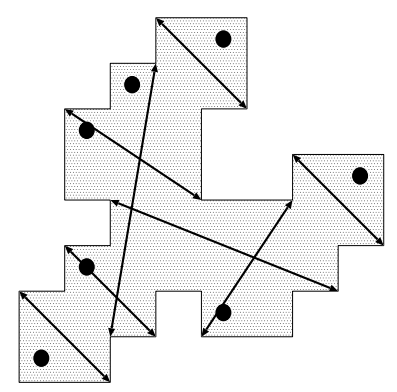

Fig. 30. An orthogonal polygon such that the covering number is strictly greater than the independence number. 


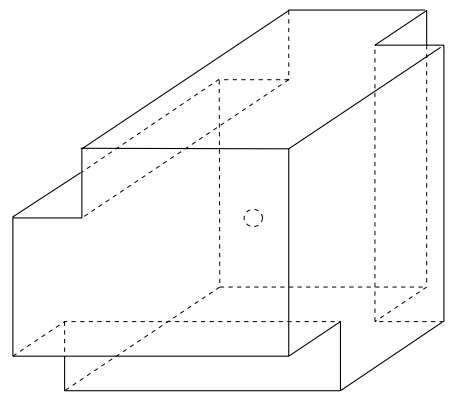

Fig. 31. The case of the three-dimensional space.

rectangle cover problem. See Section 1 and [1]. By checking the example due to Chung which does not satisfy equality between the two numbers, we see that equality does not hold for this problem, too. See Fig. 30.

Diagonal boxes are naturally defined from two vertices in the three-dimensional space, too. However, this covering problem cannot be extend to the three-dimensional case. See Fig. 31. It is easily seen that the center point cannot be covered with any diagonal box.

\section{References}

1. C. Berge, C.C. Chen, V. Chvátal, and C.S. Seow, Combinatorial properties of polyominoes, Combinatorica 1, 217-224 (1981)

2. S. Chaiken, D.J. Kleitman, M. Saks, and J. Shearer, Covering regions by rectangles, SIAM J. Algebraic Discrete Methods 2, 394-410 (1981).

3. J.C. Culberson and R.A. Reckhow, Covering polygon is hard, J. Algorithms 17, 2-44 (1994).

4. J.E. Goodman and J. O'Rourke, editors, Handbook of Discrete and Computational Geometry, CRC Press, New York, Chapters 23 (Polygons by S. Suri), 25 (Visibility by J. O'Rourke), and 43 (Pattern recognition by J. O'Rourke and S.T. Toussaint), 1997.

5. E. Győri, A minimax theorem on intervals, J. Combin. Theory Ser. B 37, 1-9 (1984).

6. E. Győri, A short proof of the rectilinear art gallery theorem, SIAM J. Algebraic Discrete Methods 7, 452-454 (1986)

7. J. Kahn, M. Klawe, and D. Kleitman, Traditional galleries require fewer watchmen, SIAM J. Algebraic Discrete Methods 4, 194-206 (1983).

8. J.M. Keil, Covering orthogonal polygons with non-piercing rectangles, Internat. J. Comput. Geom. Appl. 7(5), 473-484, (1997).

9. W.J. Masek, Some NP-complete set covering problems, unpublished manuscript, MIT, Aug. 1979 (referenced in M.R. Garey and D.S. Johnson, Computers and Intractability: A Guide to the Theory of NPCompleteness, Freeman, San Francisco, p. 232, 1979.

10. J. O'Rourke, Art Gallery Theorems and Algorithms, Oxford University Press, Oxford, 1987.

11. Mamoru Watanabe, contributed lecture at the 16th British Combinatorial Conference, 1997.

Received July 25, 1999, and in revised form March 1, 2000. Online publication May 16, 2000. 